This document is the unedited Author's version of a Submitted Work that was subsequently accepted for publication in Environmental Science and Technology, copyright (c) American Chemical Society after peer review. To access the final edited and published work see https://pubs.acs.org/doi/10.1021/acs.est.8b04752 


\section{DRomics: a turnkey tool to support the use of the dose-response framework for omics data in ecological risk assessment}

Floriane Larras $^{1 *}$, Elise Billoir ${ }^{2}$, Vincent Baillard ${ }^{2}$, Aurélie Siberchicot ${ }^{3}$, Stefan Scholz $^{1}$, Tesfaye Wubet ${ }^{4,5}$, Mika Tarkka, ${ }^{5,6}$ Mechthild Schmitt-Jansen ${ }^{1 *}$, Marie-Laure Delignette-Muller ${ }^{3}$

${ }^{1}$ Helmholtz-Centre for Environmental Research UFZ, Department of Bioanalytical Ecotoxicology, Permoserstrasse 15, 04318 Leipzig, Germany

${ }^{2}$ Université de Lorraine, CNRS, UMR 7360, LIEC, Laboratoire Interdisciplinaire des Environnements Continentaux, 57070 Metz, France

${ }^{3}$ Université de Lyon, Université Lyon 1, CNRS, VetAgro Sup, UMR 5558, Laboratoire de Biométrie et Biologie Evolutive, 69622 Villeurbanne, France

${ }^{4}$ Department of Community Ecology, Helmholtz-Centre for Environmental Research - UFZ, TheodorLieser-Straße 4, 06120 Halle, Germany

${ }^{5}$ German Centre for Integrative Biodiversity Research (iDiv) Halle-Jena-Leipzig, Deutscher Platz 5e, 04103 Leipzig, Germany

${ }^{6}$ Department of Soil Ecology, Helmholtz-Centre for Environmental Research - UFZ, Theodor-LieserStraße 4, 06120 Halle, Germany 
TOC

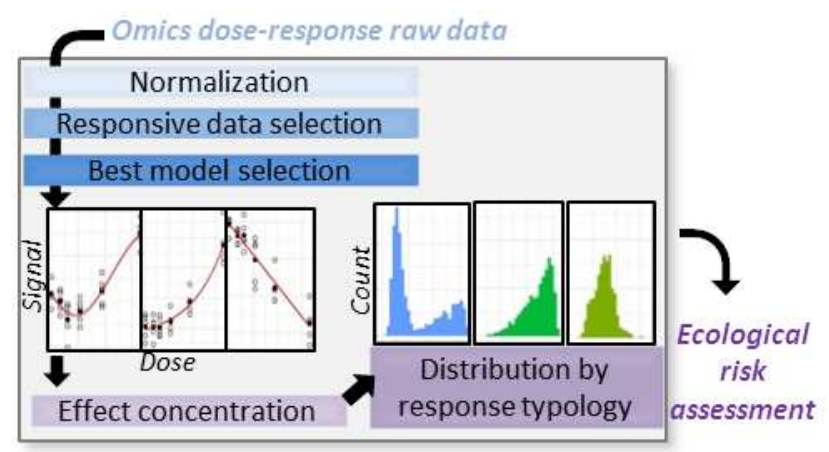

\section{ABSTRACT}

Omics approaches (e.g. transcriptomics, metabolomics) are promising for ecological risk assessment (ERA) since they provide mechanistic information and early warning signals. A crucial step in the analysis of omics data is the modelling of concentration-dependency which may have different trends including monotonic (e.g. linear, exponential) or biphasic (e.g. U shape, bell shape) forms. The diversity of responses raises challenges concerning detection and modelling of significant responses and effect concentration (EC) derivation. Furthermore, handling high-throughput datasets is time-consuming and requires effective and automated processing routines. Thus, we developed an open source tool (DRomics, available as an R-package and as a web-based service) which, after elimination of molecular responses (e.g. gene expressions from microarrays) with no concentration-dependency and/or high variability, identifies the best model for concentration-response curve description. Subsequently, an EC (e.g. a benchmark dose) is estimated from each curve and curves are classified based on their model parameters. This tool is especially dedicated to manage data obtained from an experimental design favoring a great number of tested doses rather than a great number of replicates and also to handle properly monotonic and biphasic trends. The tool finally restitutes a table of results that can be directly used to perform ERA approaches. 


\section{INTRODUCTION}

Ecological risk assessment (ERA) is defined as a procedure which estimates the probability that one or several stressors induce ecological adverse effects ${ }^{1}$, and which aims to support decision making ${ }^{2}$. ERA mainly relies on the estimation of effect concentration (EC, e.g. ECx, the concentration which affects an endpoint at x percent of the maximal effect, or BMD, the benchmark dose or concentration) obtained from concentration-response curves (CRC). Subsequently, the ECs can be used for further assessments such as Risk Quotients (PEC/PNEC) ${ }^{3}$ or sensitivity distributions ${ }^{4}$. Deriving ECs from raw data constitutes a critical step which requires dedicated approaches. Before the recent rise of omics (e.g. genomics, transcriptomics), ERA was mainly performed on the response of apical endpoints (e.g. mortality, growth, photosynthesis inhibition). Those endpoints mostly follow a sigmoidal CRC and appropriate approaches (PROAST software: www.rivm.nl/proast, DRC package ${ }^{5}$ ) were developed to model such responses and calculate ECs. In the recent years, toxicogenomics approaches were established to support the ERAprocesses. The data obtained from such approaches showed that the sigmoidal trend is rather the exception than the rule ${ }^{6,7}$. Molecular responses can be monotonic but following other than sigmoidal trends or even non-monotonic. Hence, estimating ECs from omics data requires a robust tool that can automatically handle high-throughput data, capture and model various and complex trends, and calculate ECs from such complex responses. Obtaining ECs based on omics data, comparable to those obtained from apical endpoints, is a challenge.

Various attempts have been made to manage omics data in a dose-response framework by addressing the previously stated requirements ${ }^{6-12}$. These studies present interesting approaches but several crucial steps rely on methods presenting some limitations for a dose-response framework (e.g. normalization, preselection of data with concentration dependency and low variability, models) that can lead to a loss of meaningful biological information. For example, many routines begin with a step detecting the significantly responding data (e.g. genes showing a concentration-dependent change) using a one-way ANOVA-based selection or similar method ${ }^{6-8,11}$ aiming at comparing a small number of groups with many replicates in each group. Such methods are not powerful in case of a concentration-response design 
(concentration series with a small number of replicates per concentration) ${ }^{13}$. Other methods proposed for analysis of concentration-response data ${ }^{14,15}$ only consider monotonic CRCs and hence, would miss/discard molecular responses displaying common biphasic (U shape, bell shape) CRCs. Often, the CRC modelling step compares different potential models by curve fitting based on non-linear regression and the model with the best fit is selected to describe the $\mathrm{CRC}^{6-8,11}$. However, not all of the approaches allowed deriving an EC, which would represent a crucial step in case of the use of toxicogenomics data for subsequent ERA. Furthermore, the robustness of an EC derivation from such data was never studied.

The aim of this study was to develop a workflow and a tool to facilitate the use of high-throughput omics data in ERA by providing reliable ECs. This workflow was designed to handle the various steps of omics data processing in a concentration (as well as dose)-response framework and is already provided as an open source software (DRomics) via an online interface and an R package. Our objectives were to provide a tool that enables to (1) normalize data, (2) select both monotonic and biphasic responses, (3) model CRCs for significant responses and choose the best fitting model (based on a reduced, essential set of models with a limited number of parameters, but allowing to capture a great diversity of trends), (4) characterize the shape of CRCs and (5) derive an EC from each CRC whatever its shape is. The DRomics tool is inspired from a previous workflow developed by Smetanova et al. ${ }^{7}$, but proposes the improvement of steps 2 and 3 and the addition of steps 4 and 5 . We especially paid attention to the repeatability of the results to assess the robustness of the whole approach. To exemplify our approach, we worked on a microarray-based transcriptomic dataset of the green algae Scenedesmus vacuolatus exposed to triclosan.

\section{DATA}

Scenedesmus vacuolatus transcriptomics response analysis

Cultures of chlorophyte S. vacuolatus were exposed for 14 hours to five concentrations of triclosan in the range of $0.69-6.63 \mu \mathrm{g} / \mathrm{L}$ (SI section S1). After sampling, the RNA was extracted, labelled and hybridised to Agilent (Amadid \#067797, Böblingen, Germany) microarrays (SI section S1). As no 
commercial microarray was available for this species, the microarray was designed based on a former RNA sequencing (SI section S1). Briefly, 21,495 contigs (minimum length 100 bases) were selected to design an 8X60 K microarray using eArray (Agilent Technologies, Böblingen, Germany). Each contig was represented by one or two probes in the microarray and overall 18,562 probes were replicated. The final dataset contains the fluorescence value of 61,535 probes $(18,562$ probes present twice and 24,411 present only once) for 6 treatments in 5 replicates.

\section{DEVELOPMENT OF THE DROMICS TOOL}

The TOC illustrates an overview of the main steps handled by the DRomics tool. DRomics has been released as an R package (https://lbbe.univ-lyon1.fr/-DRomics-.html) and a user-friendly online application is also available (http://lbbe-shiny.univ-lyon1.fr/DRomics-shiny/). Its workflow is organized in four steps described thereafter.

\section{Step 1: Inter-array normalization}

As a first step, the $\log 2$ fluorescence values of the microarray dataset were normalized (between arrays) in order to counterbalance for differences in hybridization efficacy. The limma R package ${ }^{16}$, which is dedicated to deal with microarray data, proposes three options to normalize single-channel data using the function "normalizeBetweenArrays": quantile, scale and cyclic loess. Briefly, the quantile normalization forces the entire empirical distributions of each array to be identical while the scale method forces only the medians of each array to be the same. The cyclic loess method, which is more sophisticated and more time consuming, is based on the repetition on each pair of arrays of a loess regression between the difference of expression between the two arrays and the average expression ${ }^{17}$. We compared the three different approaches for Scenedesmus microarray data and the effect of normalization on the distributions of the gene expression values for each array (Figure S2). With the scale method high differences were observed for high fluorescence values, with some values clearly exceeding the maximum level of raw data in each array (around 20) which was considered as not acceptable given the potential impact of 
extreme values on CRC trends. The cyclic loess and the quantile methods are known to reveal similar results. However, the cyclic loess was giving a less aggressive (i.e. weaker deformation) and more robust transformation of raw data ${ }^{16,17}$, as illustrated in Figure $S 2$. The quantile method is sometimes preferred since it is less time consuming. Hence, as the normalization step was not the rate limiting step in the data analysis in comparison to the other steps of our workflow, the cyclic loess method was chosen for the following analysis. However, the DRomics tool would nevertheless offer the opportunity to apply one of the other methods.

\section{Step 2: Selection of the responsive molecular data}

Datasets obtained from omics-approaches are typically large, representing thousands of genes and not all them respond to a concentration gradient of an exposure chemical. Therefore, we implemented a filtering step which aimed to identify significantly responding genes to the stressor, in order to focus only on the genes of interest, to reduce computational effort for modelling CRCs, and as recommended by Webster et al. ${ }^{18}$ to improve the biological relevance of derived EC values. Our purpose was to provide an approach adapted to handle dose or concentration-response data which (1) does not require many replicates for each tested concentration (and hence was more powerful than the classically used ANOVAbased method ${ }^{13}$ ) and (2) is able to detect both monotonic and biphasic CRCs. Tukey et al. ${ }^{19}$ proposed a trend test to detect monotonic trends by testing the significance of a regression line linking the response to the concentration in raw scale, log-scale or rank-scale. Assuming that the tested concentrations are well placed within the design, we favored the rank scale. Furthermore, in order to extend Tukey's trend test to the detection of biphasic trends, we tested the global significance of a first order polynomial curve (regression line) and of a second order polynomial curve (quadratic curve). The 3 approaches (ANOVAtest, linear trend test and quadratic trend test) were implemented in DRomics using the limma R package and compared. A Benjamini-Hochberg correction on the p-values was applied in order to control the false discovery rate associated to such a big dataset. A default threshold value of 0.05 was fixed for the false discovery rate. 
Based on the microarray dataset consisting of 61,535 probes with 5 replicates per condition, the quadratic trend test detected the highest number of responsive probes $(n=13,182)$ compared to the ANOVA test $(n=8,755)$ and the linear trend test $(n=5,790)$ (Figure 1A). In Figure 1 the numbers of selected genes are reported in the caption. More than ninety percent of the probes which were detected by both ANOVA and linear trend test were also detected by the quadratic trend test. In contrast, the quadratic trend test allowed detecting a considerably higher number of probes if compared to the two other tests. In order to compare the three approaches in situations with a low number of replicates per condition, the same procedure was applied but on only three or two out of the total of five replicates (Figure 1B and Figure 1C). The superiority of the quadratic trend test on the two other methods clearly appears when the number of replicates is low (Figure 1B and 1C). With only 2 replicates, the ANOVA test method was unable to select any responsive probe. In the present study, the preselection of genes responsive to triclosan was conducted based on the quadratic model alone for the following analysis, but the DRomics tool proposes the three described methods.

\section{Step 3: Response modelling and selection of the best model}

In order to select appropriate approaches for CRC modelling, we first visually examined a random set of probes (ca. a thousand) in order to detect the major trend models. Considering monotonic CRCs, we found only a minority of increasing or decreasing sigmoid curves that could be described using the classical Hill model (Table 1 and Figure 2, panels H.inc and H.dec). We did not consider any other sigmoid model in the set of models as it was already demonstrated that the different models result in very similar fits ${ }^{7,20}$ and that the choice of the sigmoid model had not a great impact on the determination of $\mathrm{ECs}^{21}$. The other observed monotonic trends were increasing or decreasing linear curves (Figure 2, panels L.inc and L.dec - simply described by a linear model) or increasing or decreasing curves with an asymptote only at low (Figure 2, panels E.inc.convex and E.dec.concave) or high concentrations (Figure 2, panels E.inc.concave and E.dec.convex). We chose to describe those four trends by the exponential 
model (Table 1). Depending of the signs of its parameters $b$ and $e$ this model is able to describe the four observed trends with a flexible curvature controlled by both parameters $b$ and $e$.

For biphasic CRCs, our objective was to be able to describe all type of observed biphasic trends in a robust way, with models strictly increasing (or descreasing, respectively) before reaching the maximal (resp. minimal) signal and then strictly decreasing (or increasing, respectively) (Figure 2 panels GP.bell and IGP.bell and GP.U and IGP.U, respectively). We also wanted to be able to describe asymptotes at low and/or high concentrations with potentially different levels for both asymptotes (Figure 2 panel GP.bell). For modelling of biphasic concentration-response curves many complex models were already proposed, usually outside the context of omics data and often associated with limitations. This is due to their complexity and too high flexibility, indicating a need to develop new mathematical models ${ }^{22}$. In the context of omics data, mainly second and third order polynomial models and Gaussian type models were proposed to describe biphasic trends. We used a Gaussian type model since polynomial models cannot describe asymptotes. Moreover, third polynomial models have more than two phases and may thus take unwanted forms (non-biphasic). However, available Gaussian models have the same asymptotic levels at low and at high concentrations. Therefore, two new 5-parameter models (Gaussian-probit and logGaussian-probit models, Table 1) were built by addition of a probit component with two asymptotic levels $d$ and $c$ to a Gaussian part (in log-scale for the log-Gaussian-probit model) of amplitude $f$, with parameters $b$ and $e$ shared by both parts (see a schematic description of the Gaussian-probit model in Figure S3). For equal $c$ and $d$ values those two models respectively correspond to classical 4-parameter Gaussian and log-Gaussian models as used in Smetanova et al. ${ }^{7}$. For different $c$ and $d$ values they provide a simple extension enabling two different asymptotic levels as often encountered in our data set as in Figure 2, panels GP.U, GP.bell, lGP.U). As done by Smetanova et al. ${ }^{7}$, we used two versions of this new Gaussian-probit model, for raw or log-scale data, in order to be able to describe symmetrical (models in raw scale) or asymetrical (models in log scale) shapes of curves around the local extremum.

Models were fitted to each previously selected probe by nonlinear regression using the $\mathrm{R}$ function "nls". Gaussian-probit and log-Gaussian-probit models were fitted both in their complete form with 5 
parameters and in their simplified form with 4 parameters (with $d=c$ ). The best-fit model was identified by the lowest Akaike Information Criterion (AIC). Probes for which the AIC value of the best model was not lower than the AIC value of the null model (constant model) minus 2 were considered as not exhibiting a concentration dependent trend and were eliminated from the probe set that was subjected to CRC modelling, as recommended by EFSA Scientific Committee ${ }^{12}$. Another filter was implemented to eliminate fits considered as not reliable. The filter was based on the test of global significance of a quadratic trend of the residuals as a function of the tested concentrations in rank-scale (similarly to the quadratic test used for the selection of responsive probes). If the trend on residuals is significant the model is not considered as reliably fitting the data and the probe is eliminated. On the 13,182 initially fitted CRCs, $1,116(8.5 \%)$ failed to pass this significance test. Those 1,116 eliminated probes mainly correspond to very noisy data sets that would have been eliminated by the selection step if the false discovery rate was fixed to a smaller value ( 0.01 or 0.001 instead of 0.05$)$ and to data sets for which the significant response was observed only at the highest tested concentration (two typical examples are given in Figure S4). In the latter case an exponential model could theoretically describe the curve, but there is not enough information in the data to estimate its curvature.

For the other 12,066 fitted CRCs, the Gaussian-probit model was chosen as the best fit model for $29.3 \%$ of the CRCs, followed by the linear model (26.5\%), the exponential model (28.1\%), the logGaussian-probit model (14.6\%) and the Hill model (1.5\%). The distribution of those fits among the 12 shapes described in Figure 2 (and supported by the models in the Table 1) is reported in the legend of Figure 2. From this classification in 12 shapes, the CRCs can be classified in four rough trends, increasing (30.7\%), decreasing (25.4\%), U-shape (24.7\%) and bell-shape (19.2\%) curves. The visual examination of each curve showed a good fit of the best-fit model. The fit is reported in Figure S5 for the first 49 selected probes (those with the most significant response).

\section{Step 4: EC calculation}


In this part, our objective was to define ECs consistent and commensurable for all previously selected probes accounting for all type of CRCs (sigmoidal, linear, exponential, biphasic (U or bell shape)).

Effective Concentrations (which affect the endpoint at $\mathrm{x} \%$ of the maximal effect, $\mathrm{ECx}$ ) have now largely supplanted No Observed Effect Concentrations (NOEC), after decades of statistical criticisms towards the latter ${ }^{23}$. ECx has a simple definition which sounds unambiguous. However, depending on the CRC shape, its derivation is not trivial and must be paid attention. When using a sigmoidal CRC, the ECx is calculated as the concentration leading to $\mathrm{x} \%$ of effect compared to the control and relatively to the amplitude of the response level between control and highest concentrations. For example, the EC50 in a Hill/log-logistic model is the abscissa of the inflection point of the CRC, but it is does not necessarily corresponds to half the control response level, unless the response level tends to zero at very high exposure concentrations. The derivation of an ECx does make sense only if an asymptotic response is observed at high exposure concentrations. For non-sigmoidal CRCs, as observed for gene expression, the calculation of such ECx is not possible and hence not suitable.

Alternatively to ECx, the Benchmark Dose (BMD) has been proposed in the field of toxicology for setting ECs ${ }^{24}$. The BMD approach described in the EFSA guidance ${ }^{12}$ proposes two options. The first option considers an $\mathrm{x}$-fold change of the control response which makes it equivalent to a $\mathrm{x} \%$ inhibition/enhancement concentration. The so-called BMD-xfold was calculated as the concentration corresponding to a Benchmark Response (BMR-xfold) defined as follows: BMR-xfold $=\mathrm{y} 0+/-\mathrm{y} 0 * \mathrm{x} / 100$, where $\mathrm{y} 0$ is the mean control response and $\mathrm{x}$ is the percentage of change. However, such BMD-xfold seems hazardously sensitive to the signal level: if the control response is zero or a very low value, so will be the $\mathrm{x}$-fold change. The BMD-xfold approach will lead to ECs closer to the control in contrast to cases with similar responses but high control levels. This is illustrated in Figure S6: both probes were described by the same CRC (linear with almost the same slope) whereas the BMD-10\%fold is much lower in case (A) where y0 has a low value, than in case (B) where y0 has a higher value. The second option defines a 
critical response level accounting for the standard deviation of data around the mean CRC. This feature makes it more robust and usable whatever the CRC model chosen, hence this method was favored in our study. The so-called BMD-zSD detects the concentration leading to a level of change compared to the control response that takes data variability around the modelled curve into account. It was calculated as the concentration corresponding to a Benchmark Response (BMR-zSD) defined as follows: BMR-zSD = $\mathrm{y} 0+/-\mathrm{z} * \mathrm{SD}$, where $\mathrm{y} 0$ is the mean control response, $\mathrm{SD}$ is the residual standard deviation of the considered CRC and $\mathrm{z}$ is the factor of SD. For linear, exponential and Hill CRCs, the BMD-xfold/BMDzSD can be analytically calculated by inversing model equations (Table 1). For biphasic CRCs, there may be two concentrations corresponding to the BMR. The BMD-xfold/BMD-zSD was defined as the lowest concentration corresponding to the BMR and it was sought numerically using the "uniroot" R function, first in the first increasing (resp. decreasing) phase and if not found in the first phase it was sought in the second decreasing (resp. increasing) phase. Both situations are illustrated in Figure S7.

BMD-zSD for $\mathrm{z}=1$ (value proposed in EFSA guidance ${ }^{12}$ ) and $\mathrm{z}=2$ and BMD-xfold for $\mathrm{x}=5,10$ and $20 \%$ were calculated for every fitted CRC. In some cases the BMD cannot be estimated because it was beyond the tested concentration range or because the BMR stood outside the range of response values defined by the model. Hence BMD-5\%fold (resp. -10\%fold and -20\% fold) could not be determined for 7.2\% (resp. $34.1 \%$ and $65.7 \%$ ) of fitted CRCs and BMD-1SD (resp. -2SD) could not be determined for 0.5\% (resp. $37.3 \%$ ) of fitted CRCs. On our data set, BMD-1SD corresponded to an average xfold change of $8.7 \%$ (interquartile range $3.4-11.4] \%$ ) (min-max [0.8-63.8]\%), BMD-2SD to an average xfold change of $14.3 \%$ (interquartile range [5.9-17.4]\%) (min-max [1.6-127.7]\%). The distribution of all of the BMD-1SD values (as provided by the tool) is globally represented in Figure 3 and by model typology in Figure S8. Given the shape of models (Figure 2), we logically obtained high BMD-1SD values for decreasing concave and increasing convex exponential CRCs and low BMD-1SD values for decreasing convex and increasing concave exponential CRCs. Log-Gauss CRCs mostly corresponded to low BMD-1SD values, 93.1\% of them being below the extremum For Gauss CRCs, $63.5 \%$ of BMD-1SD are below the extremum, which explain the observed bimodal distribution of BMDs. For Hill models, we also observed two modes, hard 
to visualize in the Figure because of a low occurrence. The second mode, about $6 \mu \mathrm{g} / \mathrm{L}$, probably corresponded to Hill CRCs where the model fitting was mostly driven by the response at the highest exposure concentration $(6.63 \mu \mathrm{g} / \mathrm{L})$. For linear models, BMD-1SD distributions were bell-shaped and spread across the tested concentration range.

\section{Repeatability of the results}

In the studied data set, 18,562 probes were represented twice on the microarray, which enabled us to analyze the repeatability of the results of the workflow by comparison of the results obtained for two same probes. Using the quadratic selection with an FDR of $0.05,71.6 \%$ of the probes selected using replicate 1 were also selected using replicate 2 of those probes. This proportion is increasing when the FDR is decreased and is stabilizing around $78 \%$ for FDR values inferior or equal to 0.001 . The two other selection methods (linear trend test and ANOVA test) gave very similar results (respectively $75 \%$ and $76 \%$ for an FDR of 0.001). Using quadratic selection and an FDR of 0.05 the proportion of probes classified in the same trend ( $\mathrm{U}$, bell, increasing, decreasing) is $89 \%$. This proportion is not much influenced by the FDR. 36\% of the observed discordances correspond to replicates classified in bell or decreasing trend depending on the replicate (example of probe "13613" in Figure S9) and 50\% of the discordances correspond to replicates classified in $\mathrm{U}$ or increasing trend depending on the replicate (example of probe "39905" in Figure S9). Strong differences between BMD values could be observed in such cases (probe "13613" and "39905” in Figure S9), but also with responses described with the same bi-phasic model: in few cases (see probe "23384" in Figure S9) the BMR can be reached in the first phase with one replicate and in the second for the other one. Globally, the Root Mean Square Error (RMSE: root mean squared differences between BMD-1SD values estimated for both replicates of each probe) was estimated at 1.198 for an FDR of 0.05 and 0.882 for an FDR of 0.001. This RMSE is increasing when the FDR is decreasing and is stabilizing below 0.9 for FDR values inferior to 0.001. For comparison, the repeatability of BMD-1SD values derivated from the same data set (replicated probes both selected with an FDR inferior to 0.001) using BMDExpress was globally poorer (RMSE of 1.215), especially for CRCs 
classified as biphasic by DRomics (RMSE of 1.803 for BMDExpress in comparison to 1.079 for DRomics) (see section S8 for details).

\section{DISCUSSION}

\section{Recommendation for experimental design}

The quality of modelling a concentration-response curve is dependent of the information provided by the data set. Replicates enable to characterize the variability around the mean concentration-response. If resources are limited, one should favor the number of concentrations rather than the number of replicates, and try to equally spread a maximal number of concentrations on the whole range of the expected response. This would not impair the precision of the residual standard deviation required for the BMDzSD calculation that only depends on the total number of points on the CRC. In our example, the design (6 concentrations with 5 replicates per concentration) was minimal to model the CRC, and it may have been advantageous to have a greater number of concentrations with two or three replicates per concentrations only. While building our workflow, we paid attention not to presuppose a great number of replicates, to ensure its applicability to typical dose-response designs. One perspective of this work will be to evaluate the impact of the number of concentrations on the workflow results, from data sets initially designed with more tested concentrations, in a purpose of design optimization.

\section{Recommendation regarding the use of the workflow}

At each step of the DRomics workflow, the user is free to choose several options (all the options tested within this work), both in the $\mathrm{R}$ package and the online version. However, we wish to provide some recommendations. For the selection step, the power of the ANOVA-based selection will increase with the number of replicates, which should not be favored in a concentration-response framework as explained before. In order to select only monotonic dose-response trends, a linear trend test may be a relevant choice. In contrast, a quadratic trend test proved efficient in our study where we wanted to select both monotonic and biphasic trends. With a very large dataset like ours (e.g. 60,000 probes), a selection of data 
(e.g. probes) is necessary not only to reduce the amount of information to be further processed (fitting and post-treatment/interpretation) but also to eliminate too noisy signals that may impair the quality and biological relevance of results ${ }^{18}$. We recommend the use of a selection step with a low FDR value (lower than 0.05 and ideally at 0.001 ) that ensures a good repeatability of the workflow results. For the choice of derived ECs, we already discussed a number of shortcomings regarding ECx (calculable only for sigmoid CRCs) and BMD-xfold which is equivalent to an ICx (hazardously sensitive to the control response level). We recommend using BMD-zSD that highlights a "critical" change compared to control, taking data variability into account. This approach has interesting features: it is calculable whatever the concentration-response shape is and does not depend on the experiment design like does the NOEC/LOEC. Instead of choosing $\mathrm{x}$ for ECx or BMD-xfold, one has to choose $\mathrm{z}$ for BMD-zSD, the number of SD considered as "critical" change. EFSA ${ }^{12}$ proposed to set $z=1$. Setting $z=2$ would define the "critical" change as the theoretical upper or lower bound of the $95 \%$ coverage interval of control data. Note that the corresponding xfold change can be calculated afterwards.

\section{Contribution of DRomics outputs for ERA process}

In ERA, OMICs approaches are rarely used in a concentration-response framework but to compare responses between 2 different conditions (e.g. upstream vs. downstream). This kind of designs present a high interest for biomarkers identification ${ }^{25}$ or environmental monitoring ${ }^{26,27}$ but used in a concentrationresponse framework, omics might also support regulatory issues (i.e. point of departure determination) and improve our mechanistical understanding regarding stressors impact on the biota. For example, some studies have already shown the interest of omics dose-responses for ERA by providing for example a metabolites effect index ${ }^{28}$ or to highlight proteins response dynamics to chemicals ${ }^{6}$. The outputs of the DRomics tool (e.g. BMD, typology, and sensitivity distribution of the probes) allow supporting those goals. The derivation of an EC from non-sigmoidal curves is also handled by other tools, such as the BMDExpress $^{8}$ (with an ANOVA-based selection step) and PROAST (without any molecular data selection step and in a far less automated workflow). In comparison to BMDExpress, DRomics is 
dedicated to typical concentration-response designs and so compatible with a low number of replicates per treatment. Moreover, DRomics ensures a more efficient selection of biphasic CRCs and a better description of them, with a non-negligible mean difference in AIC values of 3.09 (Figures S10 and S11). As a consequence, DRomics gives more repeatable results for such biphasic CRCs, and a more conservative BMD distribution (Figures S11 and S12) in comparison to BMDExpress which seems to underestimate BMD values for biphasic CRCs.

Our tool provides as an output the cumulated distribution of the BMD of the data of interest (here probes, Figure 3). This sensitivity distribution echoes the species sensitivity distribution concepts and thus exemplifies a first step (among the various possibilities of the tool) toward the use of omics data in ERA. Effect concentrations could also be a common language between biological level and omics. That might promote the establishment of links between levels and support mechanistical understanding using for example the functional annotation of the data. In this context, the tool can run on any other type of data that can be fitted directly by least-square regression and do not require a normalization step or that have been adequately processed in advance (e.g. metabolome data). Thus, DRomics provides comparable management for various omics data and facilitates multi-omics analysis. In the future, this tool will also be able to process RNA-seq data for which we plan to adapt the two first steps of the workflow taking into account the nature of RNA-seq data ${ }^{29}$. It will thus offer a simple way to compare the results obtained from different genomics platforms and evaluate their respective biological relevance as done by Webster at al. ${ }^{18}$ in toxicogenomics. The developed procedure is now available both as an $\mathrm{R}$ package named DRomics (https://lbbe.univ-lyon1.fr/-DRomics-.html) and as an online Shiny application (http://lbbeshiny.univ-lyon1.fr/DRomics-shiny/) also named DRomics based on the previous package. 


\section{FIGURES}

A

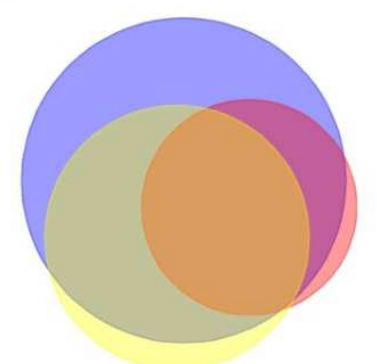

C

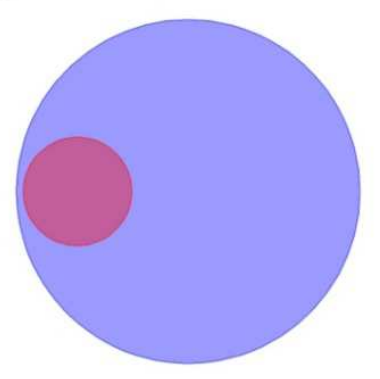

B

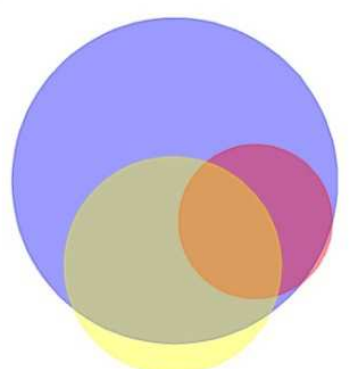

quadratic trend test

$\square$ linear trend test

ANOVA test

Figure 1. Venn diagram of the probes selected by the quadratic trend test, linear trend test and ANOVA test methods based on the datasets composed of A) 5 replicates, B) 3 replicates and C) 2 replicates. The number $\mathrm{N}$ of probes selected by the quadratic trend test (resp. linear trend test and ANOVA test) methods are A) $\mathrm{N}=13,182(\operatorname{resp} \mathrm{N}=5,790$ and $\mathrm{N}=8,755)$ with 5 replicates, $\mathrm{B}) \mathrm{N}=7,616($ resp. $\mathrm{N}=1,692$ and $\mathrm{N}=$ 3,376) with 3 replicates and C) $\mathrm{N}=593($ resp $\mathrm{N}=59$ and $\mathrm{N}=0)$ with 2 replicates. 

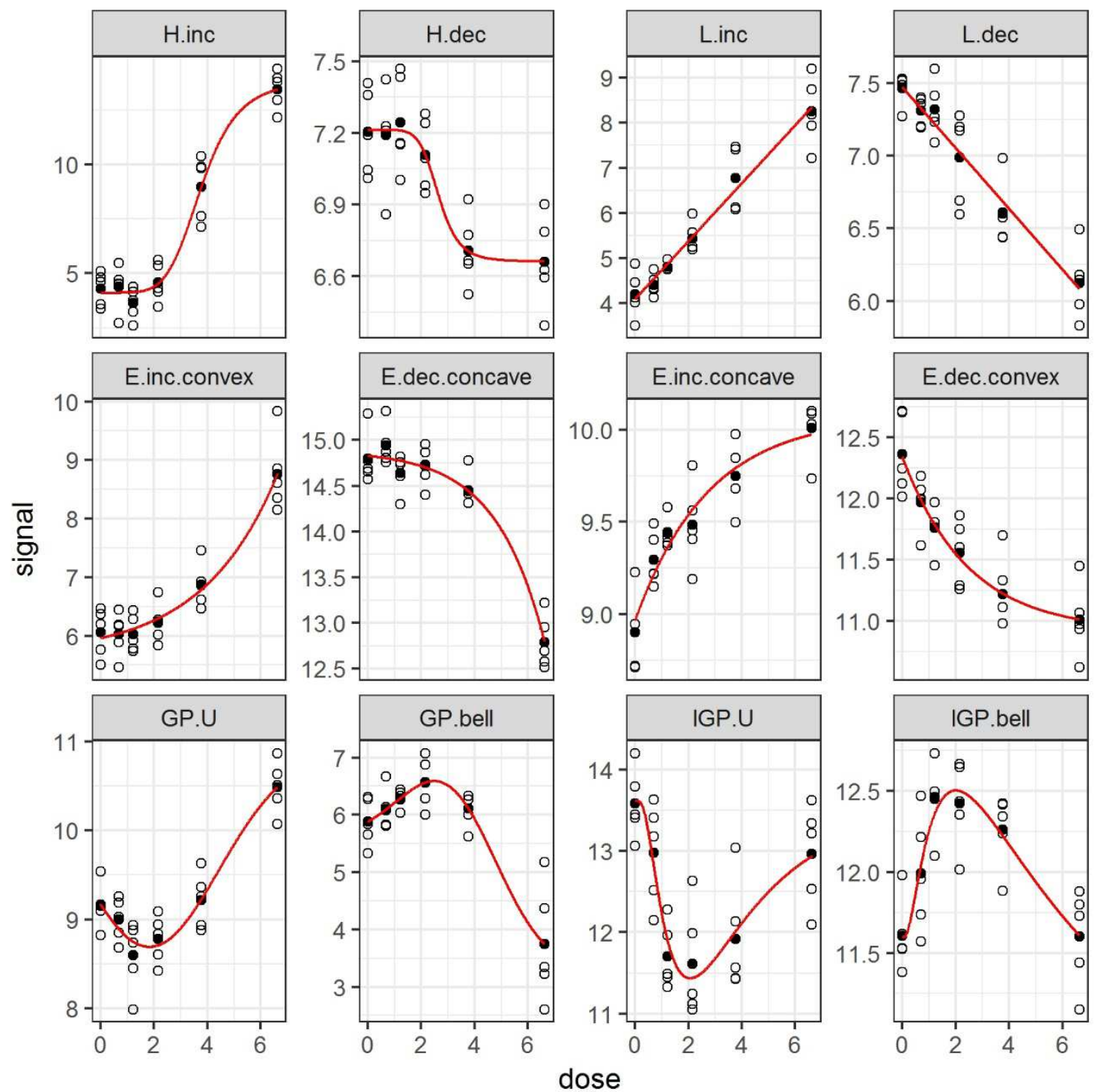

Figure 2. Illustration of various trends observed among the data set: selected data sets with corresponding best-fit models and associated percentages of CRCs for each shape : the Hill model with $d<c$ (coded H.inc for increasing - $0.8 \%$ of curves) or $d>c$ (coded H.dec for decreasing - $0.6 \%$ of curves), the linear model with $b>0$ (coded L.inc for increasing - $14.6 \%$ of curves) or $b<0$ (coded L.dec for decreasing $12.0 \%$ of curves), the exponential model with $e>0$ and $b>0$ (coded E.inc.convex for increasing convex $-11.8 \%$ of curves) or $e>0$ and $b<0$ (coded E.dec.concave for decreasing concave $-9.4 \%$ of curves) or $e<0$ and $b<0$ (coded E.inc.concave for increasing concave $-3.5 \%$ of curves) or $e<0$ and 
$b>0$ (coded E.dec.convex for decreasing convex - 3.4\% of curves), the Gaussian-probit model with $f<0$ (coded GP.U for U-shape $-16.8 \%$ of curves) or $f>0$ (coded GP.bell for bell-shape $-12.5 \%$ of curves) and the log-Gaussian-probit model with $f<0$ (coded lGP.U for U-shape - 7.9\% of curves) or $f>0$ (coded lGP.bell for bell-shape $-6.7 \%$ of curves). Fitted curves are reported in red against the observed response with replicates represented in open circles and means of replicates at each dose represented by solid circles. 


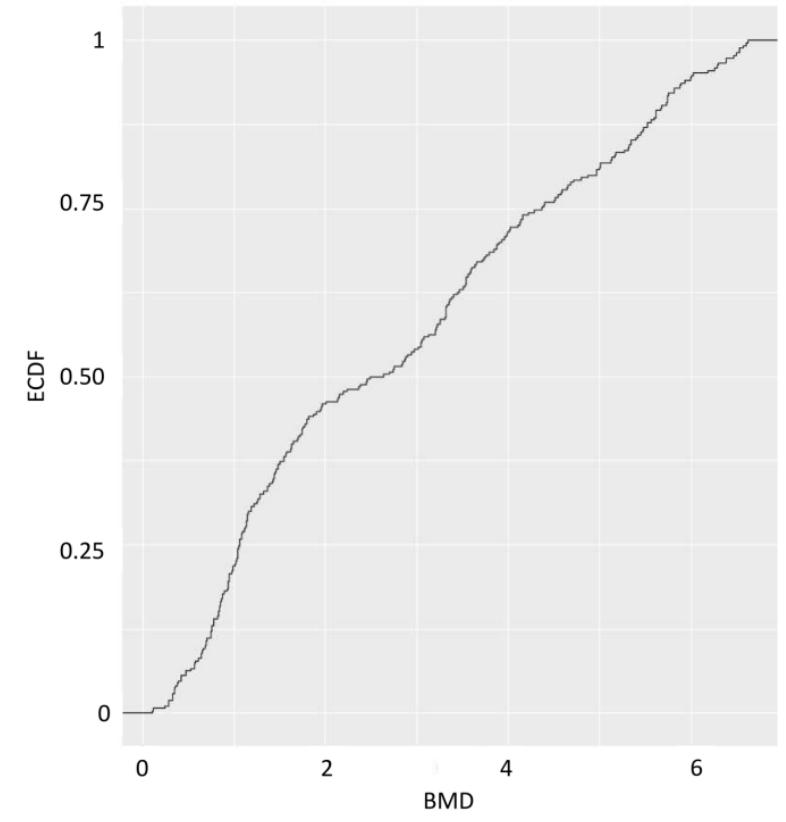

Figure 3. Cumulative distribution of probe sensitivities (BMD) provided and exported from the DRomics tool. The $\mathrm{x}$ axis represents the BMD values (here, in $\mu \mathrm{g} / \mathrm{L}$ ). The $\mathrm{y}$ axis represents the Empirical Cumulative Distribution Function (here, proportion of probes). 
1 Table 1. Set of models where x represents the concentration, y the observed signal and $\Phi$ the Cumulative Distribution Function (CDF) of the 2 standard normal distribution. 


\begin{tabular}{|c|c|c|}
\hline Name & Equation & Parameters \\
\hline linear & $y=d+b x$ & $\begin{array}{l}b \text { slope } \\
d \text { mean signal at control }\end{array}$ \\
\hline Hill & $y=c+\frac{d-c}{1+\left(\frac{x}{e}\right)^{b}}$ & $\begin{array}{l}b(>0) \text { shape parameter } \\
c \text { asymptotic signal for high concentrations } \\
d \text { mean signal at the control } \\
e \text { concentration at inflection point }(>0)\end{array}$ \\
\hline exponential & $y=d+b\left(\exp \left(\frac{x}{e}\right)-1\right)$ & $\begin{array}{l}b \text { shape parameter } \\
d \text { mean signal at the control } \\
e \text { shape parameter } \\
\text { when } e>0 \text { the CRC is increasing if } b>0 \text { (decreasing if } \\
b<0 \text { ) with no asymptote for high concentrations } \\
\text { when } e<0 \text { the CRC is increasing if } b<0 \text { (decreasing if } \\
b>0 \text { ) with an asymptote at } d-b \text { for high concentrations }\end{array}$ \\
\hline Gauss-probit & $y=f \exp \left(-0.5\left(\frac{x-e}{b}\right)^{2}\right)+d+(c-d) \Phi\left(\frac{x-e}{b}\right)$ & $\begin{array}{l}b(>0) \text { shape parameter corresponding to standard deviation of } \\
\text { the Gaussian part } \\
c \text { asymptotic signal for high concentrations } \\
d \text { asymptotic signal on the left of the CRC (reached at the } \\
\text { control only for the Log-Gauss-probit model) }\end{array}$ \\
\hline $\begin{array}{l}\text { log-Gauss- } \\
\text { probit }\end{array}$ & $y=f \exp \left(-0.5\left(\frac{\ln (x / e)}{b}\right)^{2}\right)+d+(c-d) \Phi\left(\frac{\ln (x / e)}{b}\right)$ & $\begin{array}{l}e(>0) \text { shape parameter corresponding to mean of the } \\
\text { Gaussian part (for ln } e \text { in case of the log-Gaussian-probit } \\
\text { model) }\end{array}$ \\
\hline
\end{tabular}




\begin{tabular}{|l|l|l|}
\hline & & $\begin{array}{l}f \text { amplitude of the Gaussian part (U shape if } f<0 \text { and } \\
\text { Umbrella shape if } f>0)\end{array}$ \\
\hline
\end{tabular}


4 Supporting Information. The Supporting Information is available free of charge.

5 S1) Material and methods; S2) Comparison of inter-array normalization methods; S3) Description of the

6 biphasic models; S4) Modelling complementary results; S5) BMD derivation; S6) Cumulative

7 distribution of probe sensitivities per typology; S7) Illustration of types of discordances in the

8 repeatability study; S8) Comparison of DRomics and BMDExpress results.

10 AUTHOR INFORMATION

\section{Corresponding Authors}

12 *F. Larras: floriane.larras@ufz.de

$13 *$ M. Schmitt-Jansen: mechthild.schmitt@ufz.de

\section{Funding Sources}

15 The MicroERA project has received funding from the European Union's Horizon 2020 research and

16 innovation programme under the Marie Sklodowska-Curie grant agreement No 705149.

\section{ACKNOWLEDGMENT}

18 We would like to thank Philippe Veber for his precious advices. We also thank the European Commission

19 for the financial support.

\section{ABBREVIATIONS}

21 CRC: Concentration-Response Curve; EC: Effect concentration; BMD: Benchmark Dose; ERA :

22 Ecological Risk Assessment

\section{REFERENCES}


25 (1) U.S. Environmental Protection Agency. Framework for Ecological Risk Assessment. Washington, DC: Risk Assessment Forum, U.S. Environmental Protection Agency. EPA/630/R-92/001. 1992.

(2) U.S. Environmental Protection Agency. Guidelines for Ecological Risk Assessment.EPA/630/R95/002F. U.S. Environmental Protection Agency, Risk Assessment Forum, Washington, DC

(3) Amiard, J.-C.; Amiard-Triquet, C. Conventional Risk Assessment of Environmental Contaminants. In Aquatic Ecotoxicology; Elsevier, 2015; pp 25-49.

(4) Posthuma, L.; Traas, T.; Suter II, G. General Inroduction to Species Sensitivity Distributions. In Species Sensitivity Distributions in Ecotoxicology; Boca Raton: Lewis Publishers, 2002; pp 3-10.

34 (5) Ritz, C.; Streibig, J. C. Bioassay Analysis Using R. J. Stat. Softw. 2005, 12 (5).

(6) Gündel, U.; Kalkhof, S.; Zitzkat, D.; von Bergen, M.; Altenburger, R.; Küster, E. Concentrationresponse Concept in Ecotoxicoproteomics: Effects of Different Phenanthrene Concentrations to the Zebrafish (Danio Rerio) Embryo Proteome. Ecotoxicol. Environ. Saf. 2012, 76, 11-22.

Smetanová, S.; Riedl, J.; Zitzkat, D.; Altenburger, R.; Busch, W. High-Throughput ConcentrationResponse Analysis for Omics Datasets: Concentration-Response Analysis for Omics Datasets. Environ. Toxicol. Chem. 2015, 34 (9), 2167-2180.

Yang, L.; Allen, B. C.; Thomas, R. S. BMDExpress: A Software Tool for the Benchmark Dose Analyses of Genomic Data. BMC Genomics 2007, 8 (1), 387.

(9) Thomas, R. S.; Allen, B. C.; Nong, A.; Yang, L.; Bermudez, E.; Clewell, H. J.; Andersen, M. E. A Method to Integrate Benchmark Dose Estimates with Genomic Data to Assess the Functional Effects of Chemical Exposure. Toxicol. Sci. 2007, 98 (1), 240-248.

(10) Burgoon, L. D.; Zacharewski, T. R. Automated Quantitative Dose-Response Modeling and Point of Departure Determination for Large Toxicogenomic and High-Throughput Screening Data Sets. Toxicol. Sci. 2008, 104 (2), 412-418.

(11) Chauhan, V.; Kuo, B.; McNamee, J. P.; Wilkins, R. C.; Yauk, C. L. Transcriptional Benchmark Dose Modeling: Exploring How Advances in Chemical Risk Assessment May Be Applied to the 
Radiation Field: BMD and Radiation Risk Assessment. Environ. Mol. Mutagen. 2016, 57 (8), 589604.

53

(12) EFSA Scientific Committee; Hardy, A.; Benford, D.; Halldorsson, T.; Jeger, M. J.; Knutsen, K. H.; More, S.; Mortensen, A.; Naegeli, H.; Noteborn, H.; Ockleford, C.; Ricci, A.; Rychen, G.; Silano, V.; Solecki, R.; Turck, D.; Aerts, M.; Bodin, L.; Davis, A.; Edler, L.; Gundert-Remy, U.; Sand, S.; Slob, W.; Bottex, B.; Abrahantes, J. C.; Marques, D. C.; Kass, G.; Schlatter, J. R. Update: Use of the Benchmark Dose Approach in Risk Assessment. EFSA J. 2017, 15 (1).

(13) Capizzi, T.; Survill, T. T.; Heyse, J. F.; Malani, H. An Empirical and Simulated Comparison of Some Tests for Detecting Progressiveness of Response with Increasing Doses of a Compound. Biom. J. 1992, 34 (3), 275-289.

(14) Ji, R.-R.; de Silva, H.; Jin, Y.; Bruccoleri, R. E.; Cao, J.; He, A.; Huang, W.; Kayne, P. S.; Neuhaus, I. M.; Ott, K.-H.; Penhallow, B.; Cockett, M. I.; Neubauer, M. G.; Siemers, N. O.; RossMacdonald, P.; Tucker-Kellogg, G. Transcriptional Profiling of the Dose Response: A More Powerful Approach for Characterizing Drug Activities. PLoS Comput. Biol. 2009, 5 (9), e1000512.

(15) Modeling Dose-Response Microarray Data in Early Drug Development Experiments Using R; Lin, D., Shkedy, Z., Yekutieli, D., Amaratunga, D., Bijnens, L., Eds.; Springer Berlin Heidelberg: Berlin, Heidelberg, 2012.

(16) Ritchie, M. E.; Phipson, B.; Wu, D.; Hu, Y.; Law, C. W.; Shi, W.; Smyth, G. K. Limma Powers Differential Expression Analyses for RNA-Sequencing and Microarray Studies. Nucleic Acids Res. 2015, 43 (7), e47-e47.

(17) Ballman, K. V.; Grill, D. E.; Oberg, A. L.; Therneau, T. M. Faster Cyclic Loess: Normalizing RNA Arrays via Linear Models. Bioinformatics 2004, 20 (16), 2778-2786.

(18) Webster, A. F.; Chepelev, N.; Gagné, R.; Kuo, B.; Recio, L.; Williams, A.; Yauk, C. L. Impact of Genomics Platform and Statistical Filtering on Transcriptional Benchmark Doses (BMD) and 
Multiple Approaches for Selection of Chemical Point of Departure (PoD). PLOS ONE 2015, 10 (8), e0136764.

(19) Tukey, J. W.; Ciminera, J. L.; Heyse, J. F. Testing the Statistical Certainty of a Response to Increasing Doses of a Drug. Biometrics 1985, 41 (1), 295.

(20) Forfait-Dubuc, C.; Charles, S.; Billoir, E.; Delignette-Muller, M. L. Survival Data Analyses in Ecotoxicology: Critical Effect Concentrations, Methods and Models. What Should We Use? Ecotoxicology 2012, 21 (4), 1072-1083.

(21) Van Der Hoeven, N. How to Measure No Effect. PART III: Statistical Aspects of NOEC, ECx and NEC Estimates. Environmetrics 1997, 8 (3), 255-261.

(22) Nweke, C. O.; Ogbonna, C. J. Statistical Models for Biphasic Dose-Response Relationships (Hormesis) in Toxicological Studies. Ecotoxicol. Environ. Contam. 2017, 12 (1), 39-55.

(23) Jager, T. Bad Habits Die Hard: The NOEC's Persistence Reflects Poorly on Ecotoxicology. Environ. Toxicol. Chem. 2012, 31 (2), 228-229.

(24) Haber, L. T.; Dourson, M. L.; Allen, B. C.; Hertzberg, R. C.; Parker, A.; Vincent, M. J.; Maier, A.; Boobis, A. R. Benchmark Dose (BMD) Modeling: Current Practice, Issues, and Challenges. Crit. Rev. Toxicol. 2018, 48 (5), 387-415.

(25) Moisset, S.; Tiam, S. K.; Feurtet-Mazel, A.; Morin, S.; Delmas, F.; Mazzella, N.; Gonzalez, P. Genetic and Physiological Responses of Three Freshwater Diatoms to Realistic Diuron Exposures. Environ. Sci. Pollut. Res. 2015, 22 (6), 4046-4055.

(26) Pesce, S.; Beguet, J.; Rouard, N.; Devers-Lamrani, M.; Martin-Laurent, F. Response of a DiuronDegrading Community to Diuron Exposure Assessed by Real-Time Quantitative PCR Monitoring of Phenylurea Hydrolase A and B Encoding Genes. Appl. Microbiol. Biotechnol. 2013, 97 (4), $1661-1668$.

(27) Dranguet, P.; Cosio, C.; Le Faucheur, S.; Beauvais-Flück, R.; Freiburghaus, A.; Worms, I. A. M.; Petit, B.; Civic, N.; Docquier, M.; Slaveykova, V. I. Transcriptomic Approach for Assessment of 

the Impact on Microalga and Macrophyte of in-Situ Exposure in River Sites Contaminated by Chlor-Alkali Plant Effluents. Water Res. 2017, 121, 86-94.

(28) Riedl, J.; Schreiber, R.; Otto, M.; Heilmeier, H.; Altenburger, R.; Schmitt-Jansen, M. Metabolic Effect Level Index Links Multivariate Metabolic Fingerprints to Ecotoxicological Effect Assessment. Environ. Sci. Technol. 2015, 49 (13), 8096-8104.

106

(29) Love, M. I.; Huber, W.; Anders, S. Moderated Estimation of Fold Change and Dispersion for RNA-Seq Data with DESeq2. Genome Biol. 2014, 15 (12).

108 
This document is the unedited Author's version of a Submitted Work that was subsequently accepted for publication in Environmental Science and Technology, copyright (c) American Chemical Society after peer review. To access the final edited and published work see https://pubs.acs.org/doi/10.1021/acs.est.8b04752 


\section{SUPPORTING INFORMATION}

\section{DRomics: a turnkey tool to support the use of the dose-response framework for omics data in ecological risk assessment}

Floriane Larras $^{1}{ }^{*}$, Elise Billoir ${ }^{2}$, Vincent Baillard ${ }^{2}$, Aurélie Siberchicot ${ }^{3}$, Stefan Scholz ${ }^{1}$, Tesfaye Wubet $^{4,5}$, Mika Tarkka ${ }^{5,6}$, Mechthild Schmitt-Jansen ${ }^{1} *$, Marie-Laure Delignette-Muller ${ }^{3}$

1 Helmholtz-Centre for Environmental Research UFZ, Department of Bioanalytical Ecotoxicology, Permoserstrasse 15, 04318 Leipzig, Germany

${ }^{2}$ Université de Lorraine, CNRS, UMR 7360, LIEC, Laboratoire Interdisciplinaire des Environnements Continentaux, 57070 Metz, France

${ }^{3}$ Université de Lyon, Université Lyon 1, CNRS, VetAgro Sup, UMR 5558, Laboratoire de Biométrie et Biologie Evolutive, 69622 Villeurbanne, France

${ }^{4}$ Department of Community Ecology, Helmholtz-Centre for Environmental Research - UFZ, TheodorLieser-Straße 4, 06120 Halle, Germany

${ }^{5}$ German Centre for Integrative Biodiversity Research (iDiv) Halle-Jena-Leipzig, Deutscher Platz 5e, 04103 Leipzig, Germany

${ }^{6}$ Department of Soil Ecology, Helmholtz-Centre for Environmental Research - UFZ, Theodor-LieserStraße 4, 06120 Halle, Germany

*Corresponding authors: $\underline{\text { floriane.larras@ufz.de; mechthild.schmitt@ufz.de }}$

Number of pages: 17

Number of figures: 12

Number of table: 1

S1) MATERIAL AND METHODS

S2) COMPARISON OF INTER-ARRAY NORMALIZATION METHODS

S3) DESCRIPTION OF THE BIPHASIC MODELS

S4) MODELLING COMPLEMENTARY RESULTS

S5) BMD DERIVATION

S6) CUMULATIVE DISTRIBUTION OF PROBE SENSITIVITIES PER TYPOLOGY

S7) ILLUSTRATION OF TYPES OF DISCORDANCES IN THE REPEATABILITY STUDY

S8) COMPARISON OF DROMICS AND BMDEXPRESS RESULTS 


\section{S1) MATERIAL AND METHODS}

This section introduces the methods used to design the microarray and to assess the transcriptomics response of the chlorophyte Scenedesmus vacuolatus.

\section{Microarray design}

As no commercial microarray was available for $S$. vacuolatus a microarray was designed based on a former RNA sequencing experiment. In brief, synchronized cultures of the chlorophyte were challenged by 18 treatments of different stressors such as chemicals, high ionic concentrations, heat and nutrient deficiencies. Algae from these treatments were harvested at different steps of the cell cycle by centrifugation $\left(22^{\circ} \mathrm{C}, 10 \mathrm{~min}, 3300 \mathrm{~g}\right)$. Total RNA was isolated from algal pellets using Trizol (Invitrogen Carlsbad, CA) according to the manufacturer's instruction with minor modifications. Quality and quantity of the extracted RNA were analysed by a Nanodrop Spectrophotometer (ND-1000, Peqlab, Erlangen, Germany) and an Experion ${ }^{\mathrm{TM}}$ RNA Highsens Analysis Kit (BioRad). mRNA was isolated from total RNA extracts using the Dynabeads ${ }^{\circledR}$ mRNA purification kit (ThermoFischer, Germany) according to the manufacturer's instructions resulting in $1.9 \mu \mathrm{g} \mathrm{L^{-1 }}$ pooled mRNA from all exposure conditions. Preparation of cDNA library was conducted according to the manufacturer's instruction of the Roche 454 GS-FLX Titanium platform. Briefly, $0.5 \mu \mathrm{g}$ pooled mRNA was converted to double-stranded cDNA using a cDNA synthesis system Kit and a cDNA Rapid Library Prep Kit (Roche) and sequenced afterwards using the Roche 454 GS-FLX Titanium platform. The sequencing resulted in 934,866 reads with a length of up to $400 \mathrm{bps} .97 \%$ of the reads could be assembled into 22,000 contigs using Newbler software $^{1}$. Out of this data set 21,495 contigs were selected to design a 8 X60 K microarray using eArray (Agilent Technologies, Böblingen, Germany). Remaining gaps on the array were filled randomly with already represented probes. The probes sequences and the related contigs are presented in an excel file. Genome information (cDNA sequences) have been deposited in NCBI's Sequence Read Archive and are accessible through the SRA accession number PRJNA498405 (https://www.ncbi.nlm.nih.gov/sra/PRJNA498405).

\section{S. vacuolatus exposure to triclosan and RNA extraction}

Synchronized cultures of the chlorophyte S. vacuolatus (Shih. et Krauss strain 211-15, culture collection Pringsheim (SAG Göttingen, Germany)) were grown in a sterile inorganic medium ( $\mathrm{pH} 6.4$ ) at $28^{\circ} \mathrm{C}$ by using a 14:10 h light:dark cycle ${ }^{2}$. Chlorophytes were exposed for 14 hours to seven concentrations $(0.69$, 1.22, 2.15, 3.77, 6.63, 11.65, $20.47 \mu \mathrm{g} / \mathrm{L}$ ) of triclosan (CalbioChem, Germany, CAS: 3380-34-5, purity of $99.8 \%$ ) and one solvent control (final DMSO concentration of $0.1 \%$ ) for 5 replicates in $500 \mathrm{~mL}$ flasks containing $10^{6}$ cells $/ \mathrm{mL}$. Concentrations were chosen according to pre-studies, using 7 concentrations of 
triclosan in the range of $0.69-20.5 \mu \mathrm{g} / \mathrm{L}$ covering a full concentration response curve from no inhibition to $100 \%$ inhibition of algal growth and revealing an $\mathrm{EC}_{50}$ of $6.6 \mu \mathrm{g} / \mathrm{L}$. Additionally, the quality of exposure conditions was analysed revealing a recovery of triclosan in the media of $81 \%$ during the exposure duration of $14 \mathrm{~h}$. With a focus on mode-of-action-related responses in the transcriptome at low concentrations we chose an exposure range below the $\mathrm{EC}_{50}$ value, hence the two highest exposure concentrations (11.65 and $20.47 \mu \mathrm{g} / \mathrm{L})$ were not considered for the transcriptome analysis.

Two times $80 \mathrm{~mL}$ suspensions of triclosan-exposed cultures were then harvested by centrifugation $\left(22^{\circ} \mathrm{C}\right.$, $10 \mathrm{~min}, 3300 \mathrm{~g}$ ). Culture pellets were resuspended in $500 \mu \mathrm{L}$ TRIZOL and stored at $-80^{\circ} \mathrm{C}$ until further analysis. After cell lysis and RNA extraction using a FastPrep®24 homogenizer system (Biomedicals, Santa Ana, USA) and Phase LockTM Gele Tubes (5 Prime GmbH, Hamburg, Germany), total RNA was further purified using a RNeasy®Plant Mini Kit (Quiagen, Hilden, Germany) according to the manufacturer's instructions. RNA quantity and quality were analyzed using a NanoDrop Spectrophotometer (ND-1000, Peqlab, Erlangen, Germany) revealing an absorption coefficient of $260 / 280 \mathrm{~nm}$ of 1.9 on average, which is within the quality criteria for purity of extracted RNA of $1.8-2.1$ requested by the manufacturer.

\section{Microarray analysis}

In total, 40 microarrays were used in this study. The microarrays were disposed on 5 slides, meaning that each slide was composed of 8 microarrays (Figure S1). On each slide, each microarray was associated to one of the different treatments (from the control to the highest concentration tested). Thus, each slide represented one replicate.

Fifty ng of purified RNA were labeled using the Low Input Quick Amp Labeling Kit, one color (Agilent Technologies). Finally $40 \mu \mathrm{L}$ cy3-labeled fragmented cRNA per sample were hybridized to each microarray for $17 \mathrm{~h}$ at $65^{\circ} \mathrm{C}$. Then, the 40 microarrays were washed and scanned, using an Agilent DNA Microarray scanner. Fluorescence intensities were extracted using the Agilent Feature Extraction software. One of the criteria used to assess the quality of microarray analysis was based on the spot finding at the four corners of the arrays (on image per array=40 images) and the Agilent SpikeIns concentration-response. Quality criteria were satisfying for each array (Table S1). The fluorescence data have been deposited in NCBI's Gene Expression Omnibus ${ }^{3}$ and are accessible via GEO Series accession number GSE122159 (https://www.ncbi.nlm.nih.gov/geo/query/acc.cgi?acc=GSE122159). 


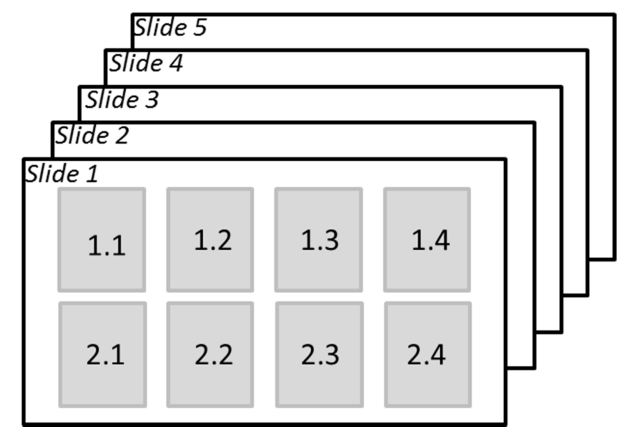

Figure S1: Microarray experimental design.

Table S1. Quality control metrics related to each microarray included in the tool development (=without 2.2 and 2.3, the two highest concentrations). For array numbers, 1.1: concentration 1; 1.2: concentration 2; 1.3: concentration 3 ; 1.4: concentration 4; 2.1 : concentration 5, 2.4: control).

\begin{tabular}{|c|c|c|c|c|}
\hline Slide & Array & Aligned to grid & SpikeIns Slope & SpikeIns $\mathbf{R}^{2}$ \\
\hline 1 & $\begin{array}{l}1.1 \\
1.2 \\
1.3 \\
1.4 \\
2.1 \\
2.4 \\
\end{array}$ & $\begin{array}{l}1 \\
1 \\
1 \\
1 \\
1 \\
1 \\
\end{array}$ & $\begin{array}{r}1.08 \\
1.05 \\
1.07 \\
1.07 \\
1.08 \\
1.1 \\
\end{array}$ & $\begin{array}{r}1 \\
0.99 \\
1 \\
0.99 \\
1 \\
0.99 \\
\end{array}$ \\
\hline 2 & $\begin{array}{l}1.1 \\
1.2 \\
1.3 \\
1.4 \\
2.1 \\
2.4 \\
\end{array}$ & $\begin{array}{l}1 \\
1 \\
1 \\
1 \\
1 \\
1 \\
\end{array}$ & $\begin{array}{l}1.07 \\
1.08 \\
1.08 \\
1.05 \\
1.09 \\
1.05 \\
\end{array}$ & $\begin{array}{r}0.99 \\
1 \\
1 \\
0.99 \\
0.99 \\
0.99 \\
\end{array}$ \\
\hline 3 & $\begin{array}{l}1.1 \\
1.2 \\
1.3 \\
1.4 \\
2.1 \\
2.4\end{array}$ & $\begin{array}{l}1 \\
1 \\
1 \\
1 \\
1 \\
1 \\
\end{array}$ & $\begin{array}{l}1.09 \\
1.07 \\
1.05 \\
1.06 \\
1.07 \\
1.09 \\
\end{array}$ & $\begin{array}{r}1 \\
0.99 \\
0.99 \\
1 \\
0.99 \\
0.99 \\
\end{array}$ \\
\hline 4 & $\begin{array}{l}1.1 \\
1.2 \\
1.3 \\
1.4 \\
2.1 \\
2.4 \\
\end{array}$ & $\begin{array}{l}1 \\
1 \\
1 \\
1 \\
1 \\
1 \\
\end{array}$ & $\begin{array}{l}1.08 \\
1.04 \\
1.08 \\
1.06 \\
1.08 \\
1.06 \\
\end{array}$ & $\begin{array}{l}1 \\
1 \\
1 \\
1 \\
1 \\
1 \\
\end{array}$ \\
\hline 5 & $\begin{array}{l}1.1 \\
1.2 \\
1.3 \\
1.4 \\
2.1 \\
2.4\end{array}$ & $\begin{array}{l}1 \\
1 \\
1 \\
1 \\
1 \\
1\end{array}$ & $\begin{array}{l}1.05 \\
1.06 \\
1.04 \\
1.06 \\
1.07 \\
1.08\end{array}$ & $\begin{array}{r}0.99 \\
0.99 \\
0.99 \\
1 \\
1 \\
1\end{array}$ \\
\hline
\end{tabular}




\section{S2) COMPARISON OF INTER-ARRAY NORMALIZATION METHODS}

Here, are presented the graphical outputs (also provided in the DRomics tool) of the normalization step for each proposed method.

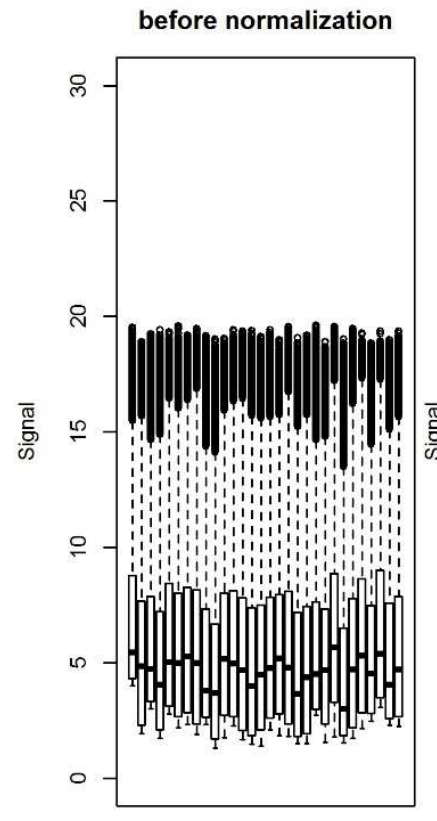

Samples

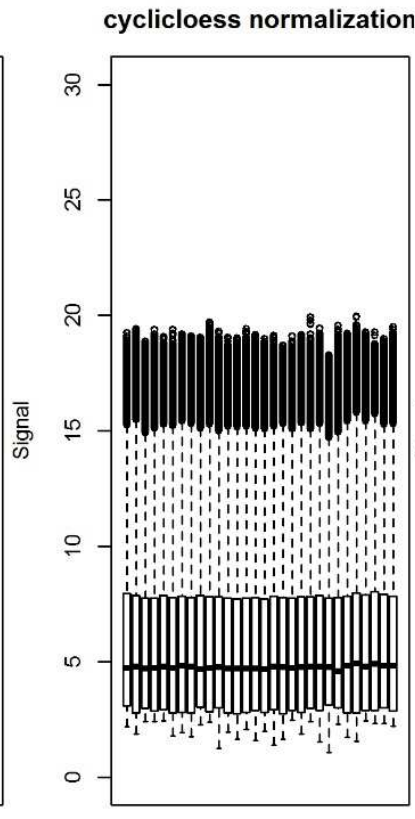

Samples
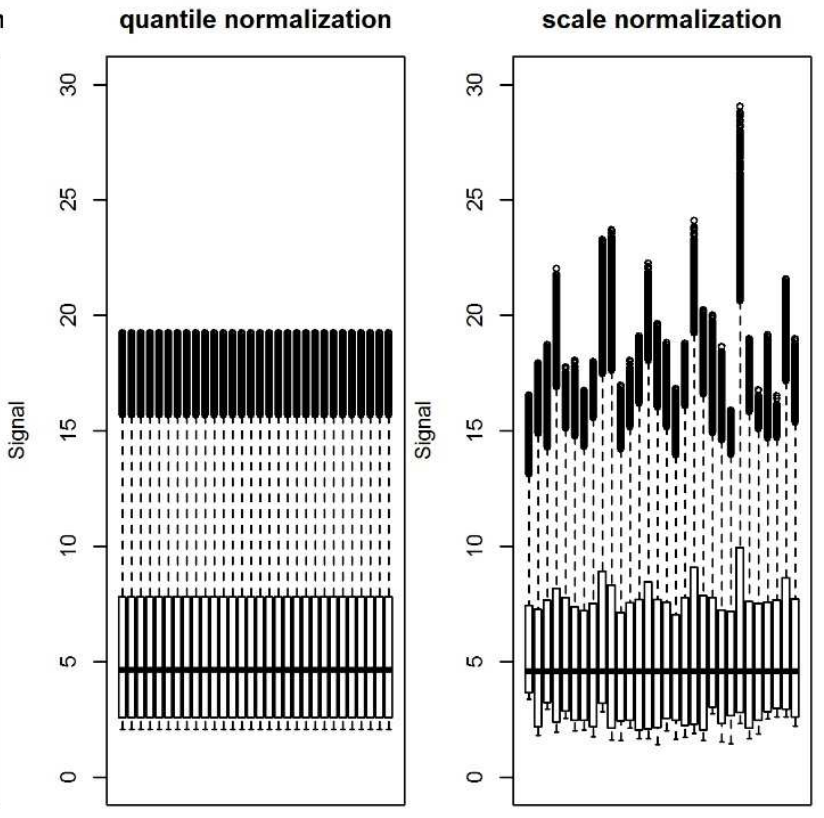

Samples

Samples

Figure S2. Boxplots of fluorescence values (in $\log 2$ ) for each array, before and after normalization by each proposed method. 


\section{S3) DESCRIPTION OF THE BIPHASIC MODELS}

\section{Description of the 5-parameter Gauss-probit and log-Gauss-probit models.}

The 5-parameter Gauss-probit model is defined as the sum of a Gauss part and a probit part as illustrated in Figure S3:

$$
y=f \exp \left(-\frac{1}{2} \times\left(\frac{x-e}{b}\right)^{2}\right)+d+(c-d) \Phi\left(\frac{x-e}{b}\right)
$$

See also Table 1 of the manuscript for parameter description.

From this formulation one can calculate its first derivative as below:

$$
\begin{gathered}
y^{\prime}=f \exp \left(-\frac{1}{2} \times\left(\frac{x-e}{b}\right)^{2}\right) \times\left(-\frac{1}{2} \times \frac{2(x-e)}{b^{2}}\right)+\frac{(c-d)}{b \sqrt{2 \pi}} \exp \left(-\frac{1}{2} \times\left(\frac{x-e}{b}\right)^{2}\right) \\
=\exp \left(-\frac{1}{2} \times\left(\frac{x-e}{b}\right)^{2}\right)\left(\frac{-f \times(x-e)}{b^{2}}+\frac{(c-d)}{b \sqrt{2 \pi}}\right) \\
=\exp \left(-\frac{1}{2} \times\left(\frac{x-e}{b}\right)^{2}\right)\left(\frac{-\sqrt{2 \pi} \times f \times(x-e)+b \times(c-d)}{b^{2} \sqrt{2 \pi}}\right)
\end{gathered}
$$

This derivative is null for a unique value of $x$ :

$$
x=e+\frac{b(c-d)}{f \sqrt{2 \pi}} .
$$

The Gauss-probit model thus describes a biphasic model if $f \neq 0$, with the maximum (if $f>0$ ) or the minimum (if $f<0$ ) reached at $x=e+\frac{b(c-d)}{f \sqrt{2 \pi}}$.

The log-Gauss-probit model is the same model in log-scale, obtained by replacing $x$ by $\ln (x)$ and $e$ by $\ln (e)$ :

$$
y=f \exp \left(-\frac{1}{2} \times\left(\frac{\ln (x)-\ln (e)}{b}\right)^{2}\right)+d+(c-d) \Phi\left(\frac{\ln (x)-\ln (e)}{b}\right)
$$

It is thus also a biphasic model if $f \neq 0$, with the maximum (if $f>0$ ) or the minimum (if $f<0$ )

reached at $\ln (x)=\ln (e)+\frac{b(c-d)}{f \sqrt{2 \pi}}$, so at $x=e \times \exp \left(\frac{b(c-d)}{f \sqrt{2 \pi}}\right)$. 


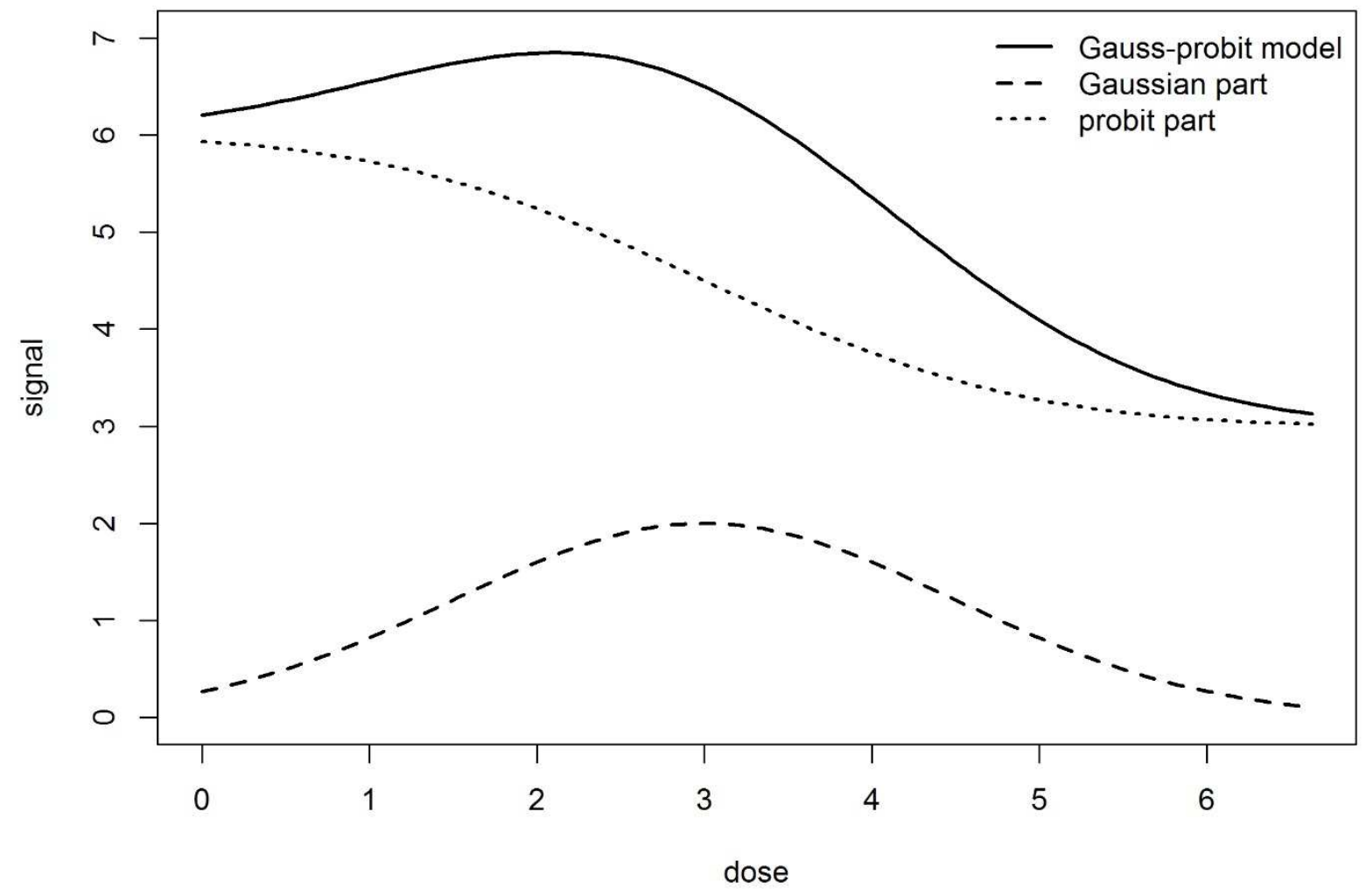

Figure S3. Schematic description of the 5-parameter Gauss-Probit model, as an addition of a Gaussian part and a probit part, with realistic values of parameters (such as some encountered in our data sets): $b=1.5, c=3, d=6, e=3, f=2$. 


\section{S4) MODELLING COMPLEMENTARY RESULTS}

This part presents complementary results for the modelling step: examples of probes first selected in the selection step and secondly eliminated in the modelling step (Figure S4) and the fit of the concentration-response curve for the first 49 probes selected (Figure S5).

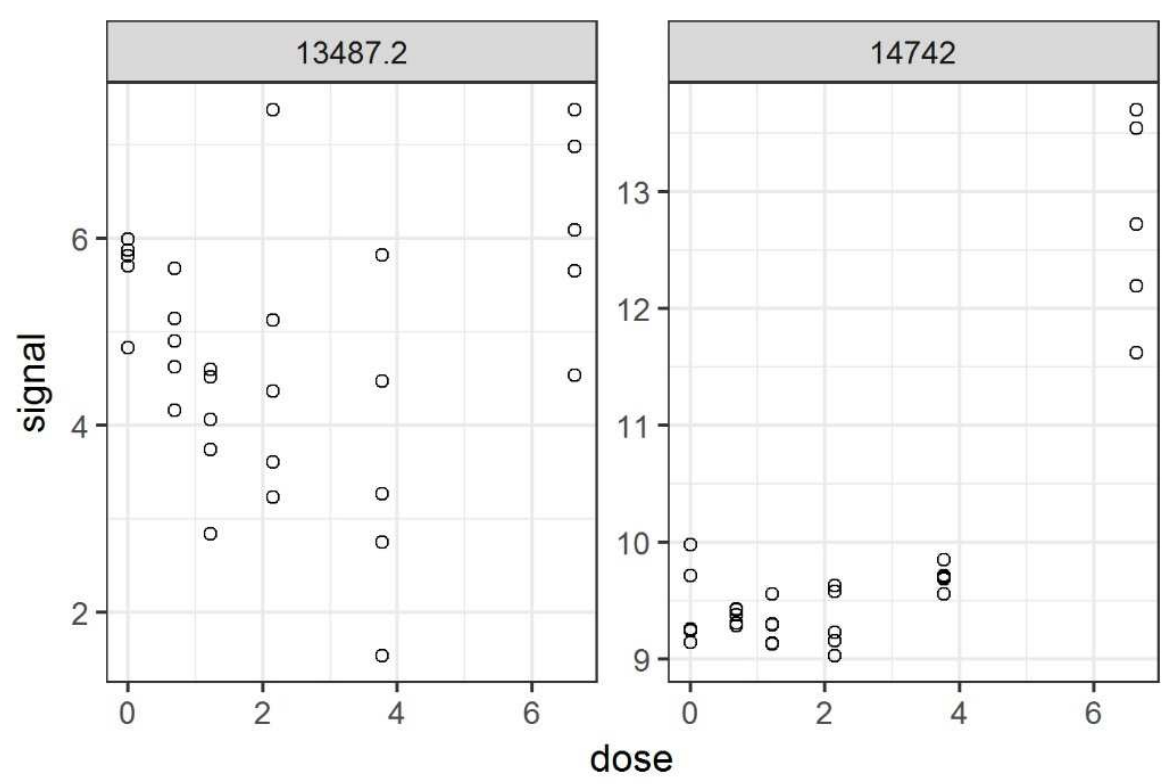

Figure S4. Typical examples of probes first selected in the selection step and secondly eliminated in the modelling step: a very noisy data set (on the left) and a data set for which the significant response was observed only at the highest tested concentration (on the right). 


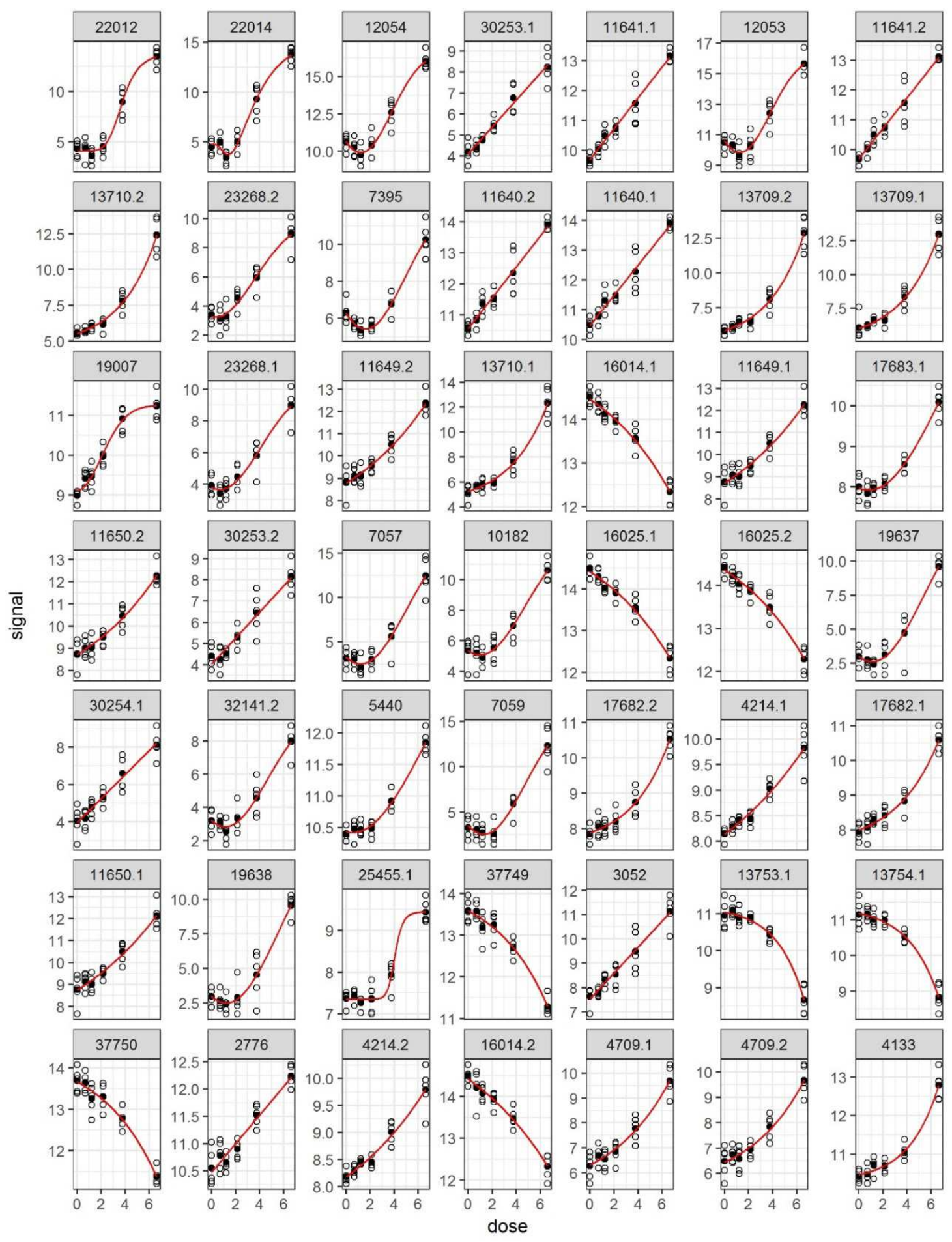

Figure S5. Fit of the concentration-response curve for the first 49 probes selected (ordered by decreasing adjusted p-values). Fitted curves are reported in red against the observed response with replicates represented in open circles and means of replicates at each dose represented by solid circles. 


\section{S5) BMD DERIVATION}

This part exemplifies how the control level influences the value of the derived BMD-xfold (Figure S6) and illustrates the calculation of the BMD on a biphasic model (Figure S7).

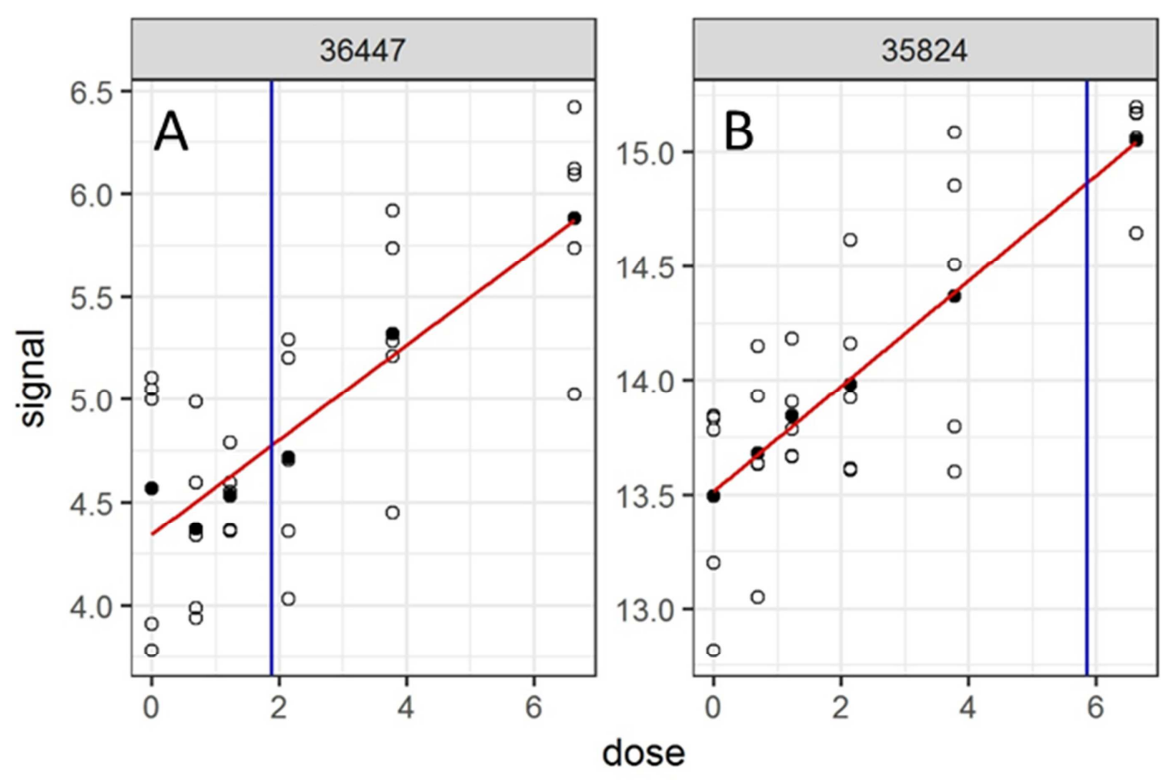

Figure S6. Illustration of BMD-10\%fold. Both probes are modelled by linear CRCs with the same slope but different intercept (y0). A situation with a low y0 value (A) leads to a lower BMD-xfold value than a situation with high y0 value (B), all other parameter remain equal. BMD-10\%fold are highlighted by blue lines. 


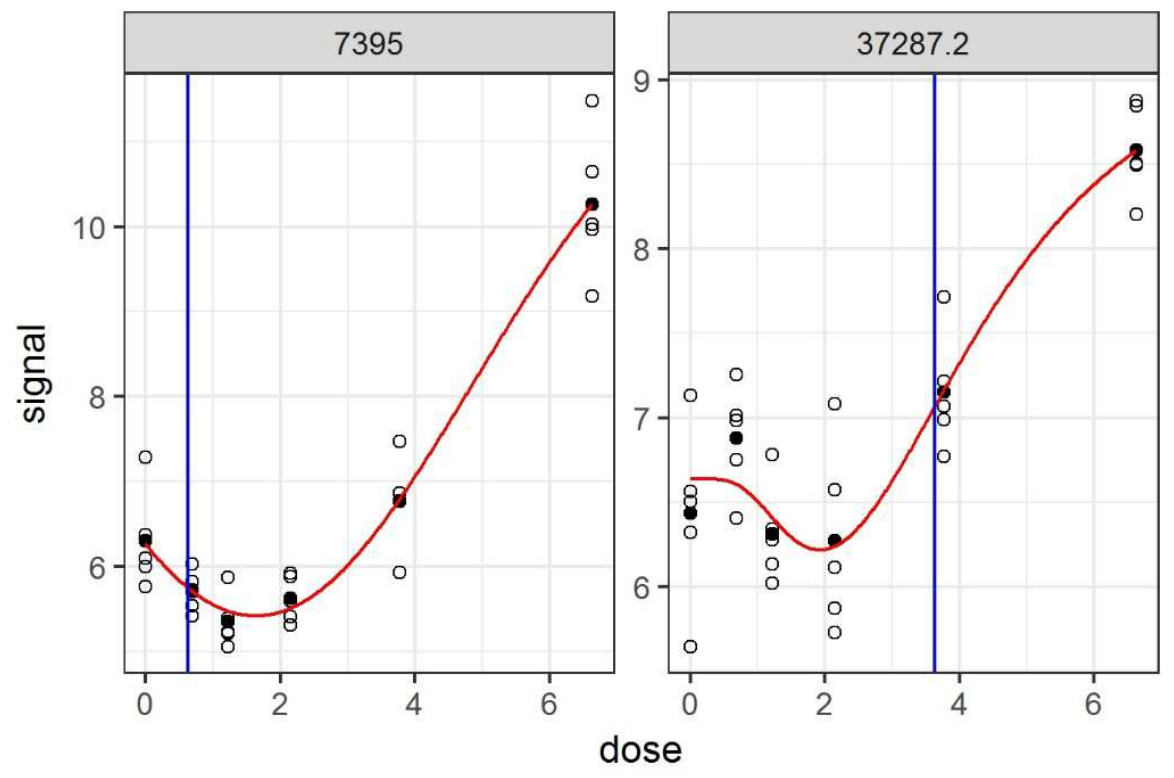

Figure S7. Illustration of situations where the BMD-1SD is found before the model extremum (on the left) and after the model extremum (on the right). BMD-1SD are highlighted by blue lines. 


\section{S6) CUMULATIVE DISTRIBUTION OF PROBE SENSITIVITIES PER TYPOLOGY}

Presentation of one of the graphical output of the DRomics tool based on the BMD values of all the probes selected by the previous steps of the workflow.
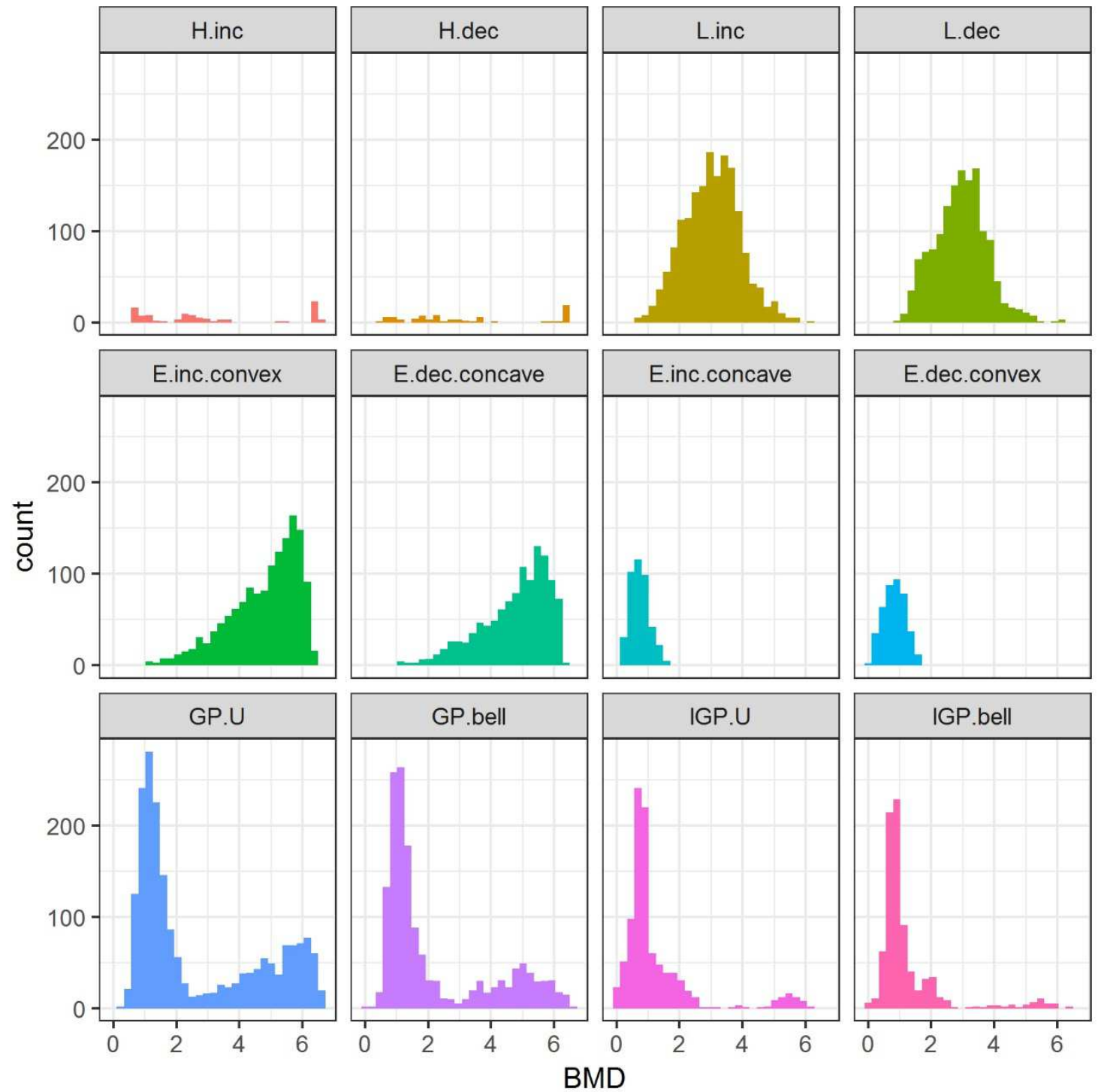

Figure S8. Distribution of the BMD-zSD values (with $\mathrm{z}=1$ ) of each probe associated specifically to each typology of curves (typology defined in the legend of Figure 2). 
The following figure gives examples of rare probes for which a great discordance was observed between both replicates.
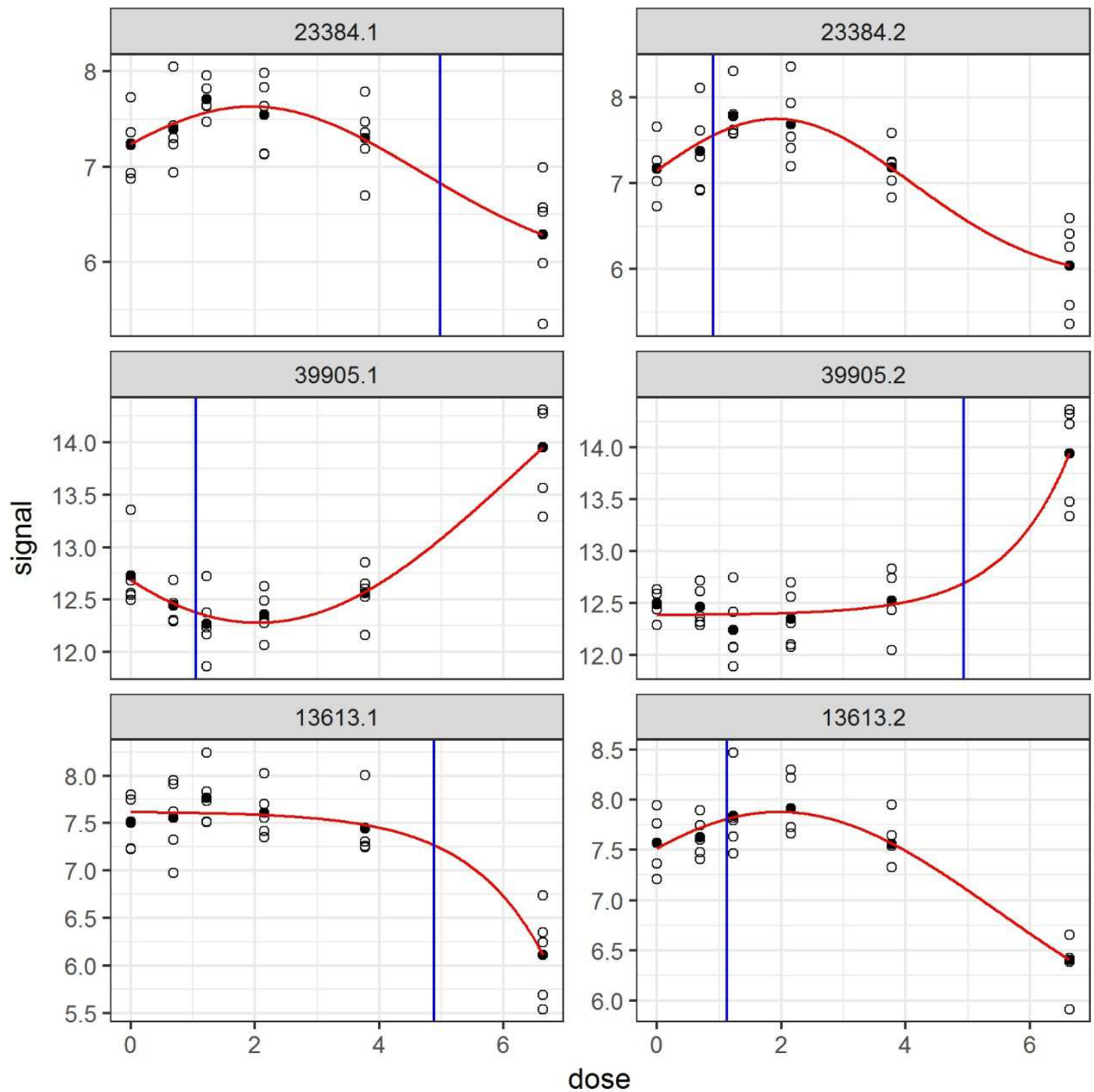

Figure S9. Examples of discordances between results for replicated probes. 


\section{S8) COMPARISON OF DROMICS AND BMDEXPRESS RESULTS}

BMDExpress is often used to model dose-response curves for omics data and to derivate BMD values. Therefore, we compared DRomics and BMDExpress results on the same data set. We especially compared their ability to describe observed responses (using AIC values), BMD values and their repeatability. For that purpose, we ran BMDExpress on a subset of 1814 probes $(=2 * 907)$, corresponding to 907 probes that were replicated and both selected using an FDR value of 0.001. For this set of samples, we used BMDExpress for the modelling and BMD calculation step. A value of a BMR of 1 SD was used in order to obtain BDM values comparable to DRomics BMD-1SD values. As BMDExpress is far more time consuming than DRomics, it is necessary using BMDExpress to properly define options to optimize the calculation on the used computer. Using a Toshiba Portege laptop (Intel Core i7 with 4 cores, 2.5 GHz, 8GB RAM, Windows 7 pro) we fixed the number of threads to 16 (four times the number of available cores on the computer, as recommended in the online tutorial) and kept the model execution time out at its default value of 600 seconds (it corresponds to the maximal time the software can run a model for an individual probe). Using those parameters, the run of BMDExpress on the subset of data took around two hours while it took around one minute using the DRomics package with the parallel computation implemented in the modelling step.

BMDExpress was used with its default choice of proposed models: linear, Hill, power, exponential and second and third order polynomial models. BMDExpress and DRomics provided the AIC value for each fitted model and the best AIC value (AIC value of the chosen model) for each CRC. As AIC values are calculated up to a constant, those AIC values were put on the same scale by equalling AIC values of the linear model given by both tools. We can see on Figure S10 that if best AIC values are rather similar for responses characterized by DRomics as monotonic ("inc" or "dec" DRomics trend), a great number of responses characterized by DRomics as biphasic ("U" or "bell" DRomics trend) are better fitted by DRomics, with a smaller AIC value. For $4.43 \%$ (resp. 0.52\%) of the probes, the best model proposed by BMDExpress revealed a higher (resp. lower) AIC than the one proposed by DRomics (absolute difference in AIC greater than 10). The mean differences of AIC values (BMDExpress - Dromics) is of 0.955, with a great heterogeneity due to trend of responses: the models chosen by BMDExpress reveal slightly lower AICs than by DRomics for monotonic responses (mean difference in AIC of - 0.38) but higher AICs for biphasic responses (mean difference in AIC of 3.09). 


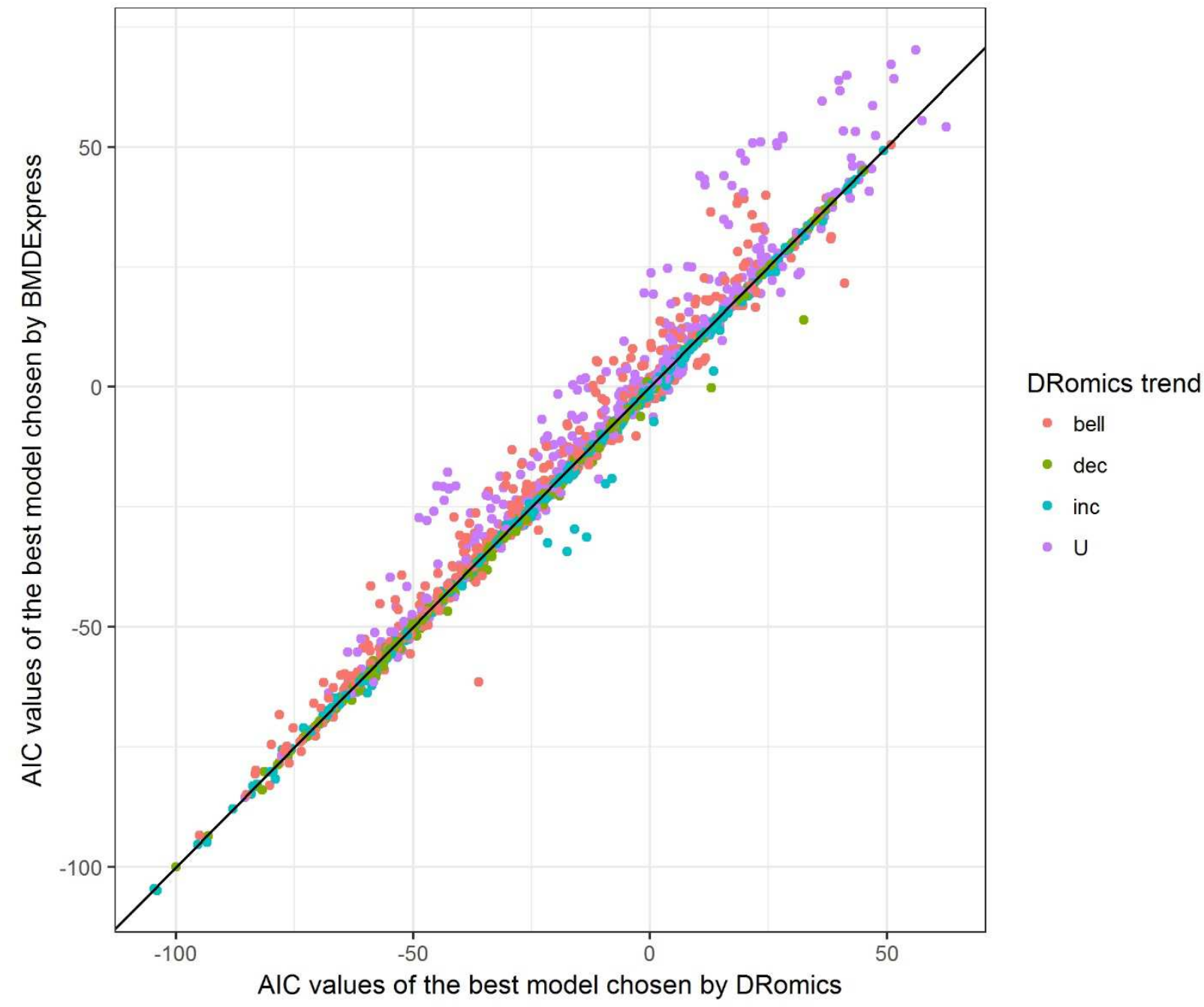

Figure S10. Scatter plot of AIC values of the best model chosen by DRomics (x-axis) and BMDExpress (y-axis), with the color of each point coding for the trend of the CRC as characterized by DRomics. 
Figure S11 corresponds to the scatter plot of BMD values estimated by DRomics (x-axis) and BMDExpress (y-axis). We can see on this plot that the tools gave well correlated values for monotonic CRCs, but not for biphasic responses, for which DRomics tends to give smaller BMD values. It seems that BMDExpress, which only proposes second and third polynomial models as non-monotonic models, has some difficulties to describe biphasic CRCs. DRomics made the choice of a biphasic model 6.4 times more than BMDExpress on this subset of data. This difficulty has a clear consequence on the BMD distribution (Figure S12) as BMDExpress may overestimates BMD values for biphasic CRCs.

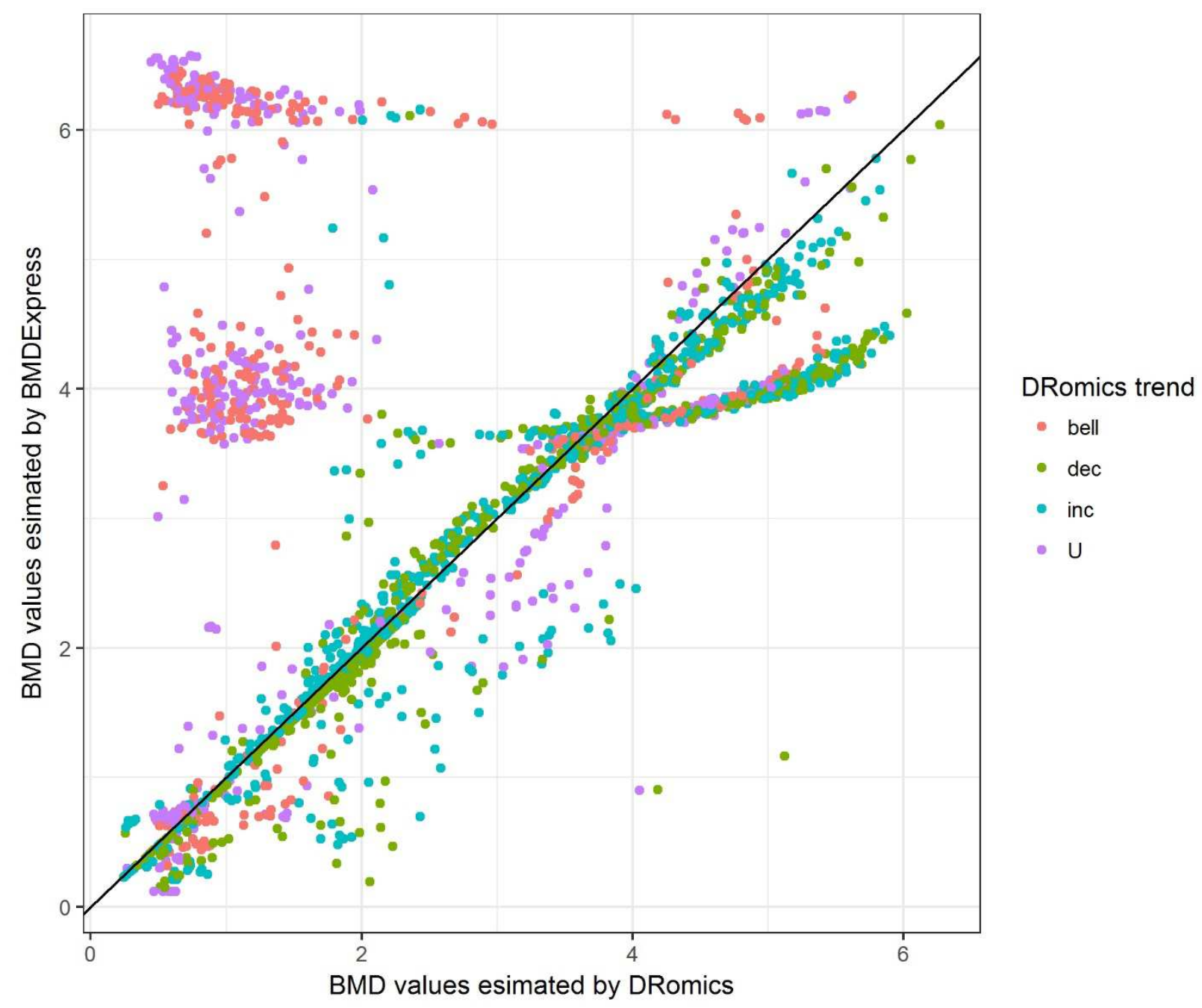

Figure S11. Scatter plot of the BMD-1SD values estimated by DRomics (x-axis) and BMDExpress (yaxis), with the color of each point coding for the trend of the CRC as characterized by DRomics. 


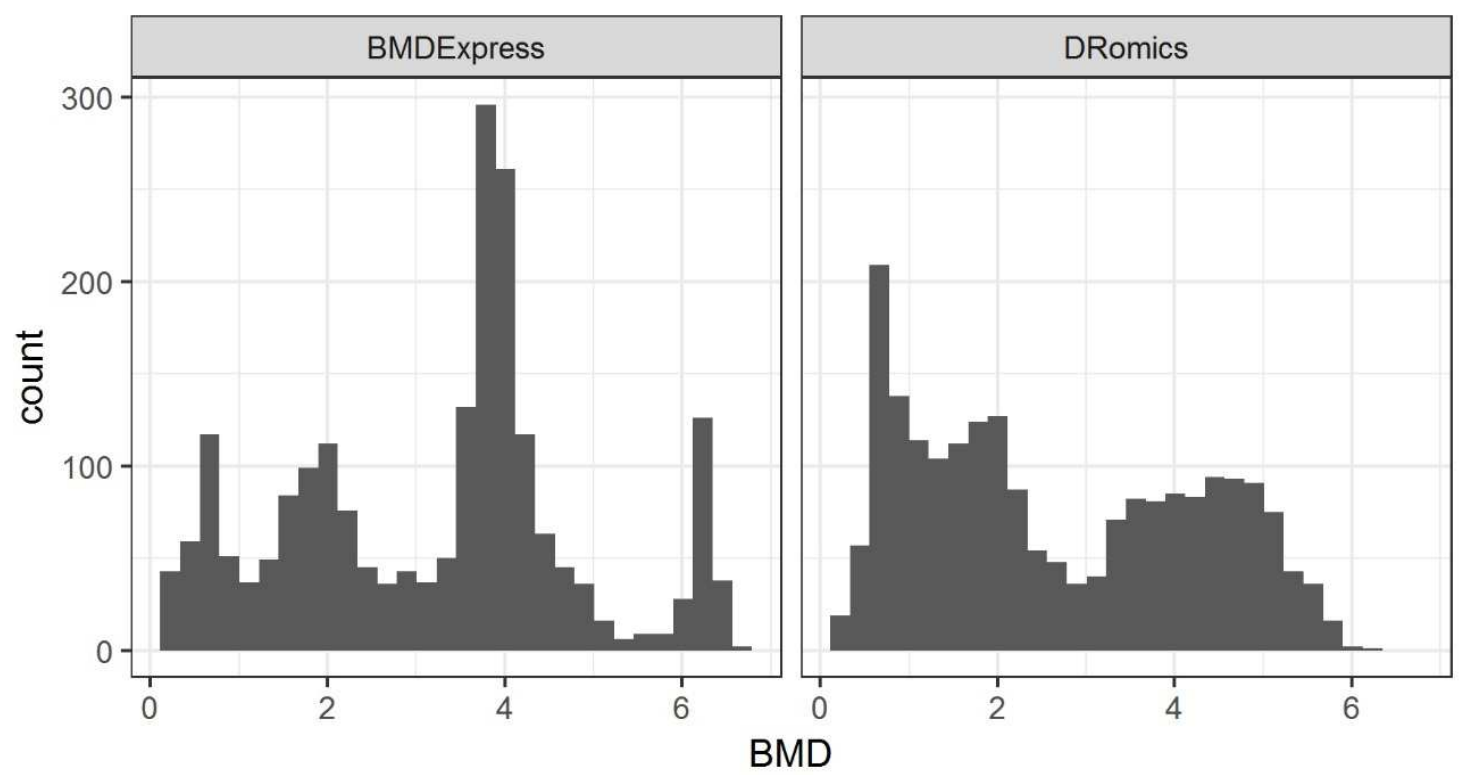

Figure S12. Distribution of the BMD-1SD values as estimated by DRomics (left) and by BMDExpress (right).

Concerning the repeatability of the results obtained per probe, the method described in part "Repeatability of the results" in the main manuscript was also applied to BMDExpress on the subset of data. The repeatability of BMDExpress was globally lower than the one of DRomics, based on values of root mean square error (RMSE: root mean squared differences between BMD-1SD values estimated for both replicates of each probe) of 0.882 for DRomics and 1.215 for BMDExpress. DRomics gave a slightly higher RMSE for biphasic responses (1.079) than for monotonic responses (0.733) while BMDExpress gave less repeatable results for probes with a biphasic response (respective RMSE values of 1.803 and 0.615). This last result confirms the difficulty to give a relevant and repeatable estimation of the BMD value for biphasic responses using BMDExpress. 


\section{REFERENCES}

(1) Kumar, S.; Blaxter, M. L. Comparing de Novo Assemblers for 454 Transcriptome Data. BMC Genomics 2010, 11 (1), 571.

(2) Altenburger, R.; Walter, H.; Grote, M. What Contributes to the Combined Effect of a Complex Mixture? Environ. Sci. Technol. 2004, 38 (23), 6353-6362.

(3) Edgar, R. Gene Expression Omnibus: NCBI Gene Expression and Hybridization Array Data Repository. Nucleic Acids Res. 2002, 30 (1), 207-210. 